\title{
Dynamics and Control of a Tethered Satellite System Based on the SDRE Method
}

\author{
Yong-Lin Kuo \\ Graduate Institute of Automation and Control, National Taiwan University of Science and Technology, No. 43, Sec. 4, Keelung Road, \\ Taipei City 10607, Taiwan \\ Correspondence should be addressed to Yong-Lin Kuo; yl_kuo@yahoo.com
}

Received 1 April 2016; Accepted 25 July 2016

Academic Editor: Anton V. Doroshin

Copyright (c) 2016 Yong-Lin Kuo. This is an open access article distributed under the Creative Commons Attribution License, which permits unrestricted use, distribution, and reproduction in any medium, provided the original work is properly cited.

\begin{abstract}
This paper presents the nonlinear dynamic modeling and control of a tethered satellite system (TSS), and the control strategy is based on the state-dependent Riccati equation (SDRE). The TSS is modeled by a two-piece dumbbell model, which leads to a set of five nonlinear coupled ordinary differential equations. Two sets of equations of motion are proposed, which are based on the first satellite and the mass center of the TSS. There are two reasons to formulate the two sets of equations. One is to facilitate their mutual comparison due to the complex formulations. The other is to provide them for different application situations. Based on the proposed models, the nonlinear dynamic analysis is performed by numerical simulations. Besides, to reduce the convergence time of the librations of the TSS, the SDRE control with a prescribed degree of stability is developed, and the illustrative examples validate the proposed approach.
\end{abstract}

\section{Introduction}

A tethered satellite system (TSS) usually consists of several spacecraft, a long tether which connects these spacecraft and a tether deployment/retrieval system. In 1895, Tsiolkovsky conceived the concept of a TSS [1]. In 1974, Colombo et al. proposed a "Shuttle-borne Skyhook" for low-orbital altitude research, which marked the advent of tethered satellite systems (TSS) [2]. Since then, there are several potential applications in space technologies, such as scientific experiments, deployment or retrieval of satellites, power generation, measurement of aerodynamic forces.

A classical TSS consists of two satellites connected by a tether, and one of the simplest models is a one-piece dumbbell, where the two satellites are assumed to be point masses and the tether is considered to be a straight massless line [3]. He et al. studied the stability of a TSS by utilizing Taylor expansion formula and stability criterion, and a rangerate control algorithm is developed to achieve the stable control of tether's deploying, keeping, and retrieving [4]. Nakanishi et al. investigated the in-plane periodic solutions for a dumbbell model in elliptic orbits using bifurcation, and their trajectories are projected on the van der Pol planes to predict when the delayed feedback control will need to act to maintain the periodic motions [5]. Kojima et al. conducted an experimental study to investigate the effect of gravity for the libration of tethered satellite system by delayed feedback control [6]. Zhang et al. presented several criteria on the existence of periodic solutions for a TSS in an elliptical orbit, and the uniqueness of periodic solutions for the TSS in a circular orbit is presented on the basis of coincidence degree theory [7]. Hu et al. presented the theoretical and experimental studies of the deployment and retrieval control of a TSS, and the ideas of online optimization and receding horizon control were applied to design a feedback controller for the TSS [8]. Jung et al. presented a dynamic analysis of a TSS with a moving mass based on a two-piece dumbbell model, where the moving mass is conveyed between two satellites along a straight massless tether [9]. The same authors analyzed the nonlinear dynamic behavior of deployment and retrieval of a three-body TSS on a variable-radius orbit, and the system is modeled as a two-piece dumbbell model with six degrees of freedom, which consists of three point masses and two straight massless tethers [10]. Hong and Varatharajoo discussed the development of mathematical models for a flexible TSS in both planar and coplanar states, where the TSS 
consists of three rigid bodies with two flexible tethers, each connecting two rigid bodies with one located in the center and serving as the mothership [11]. Hong et al. developed the analytic solutions for a TSS subjected to internal tether tension moment and external aerodynamic torque for spinup and spin-down maneuvers by the approximation of Euler's equations of motion via Fresnel integrals and sine and cosine integrals [12]. Furthermore, there are some review papers addressing the technologies and applications of a TSS [13-17].

For nonlinear optimal control, Pearson presented a linear time and state-dependent approximation to a nonlinear and nonstationary system optimized with respect to a quadratic performance index by treating it as an instantaneously linear stationary system in 1962 [18]. This approach was further investigated by Coultier et al., who extended the LQR approach to propose a so-called state-dependent Riccati equation (SDRE) method for the nonlinear quadratic regulation problems [19]. Zhao and Deng investigated the relationship between the technique by SDRE and HamiltonJacobi-Isaacs equations for nonlinear $H^{\infty}$ control design by establishing Lyapunov matrix equations for partial derivatives of the solution of the SDREs and introducing symmetry measure for some related matrices [20]. Nekoo investigated a general case which has control nonlinearities and timevarying weighting matrix $Q$ is solved with three approaches: exact solution, online control update, and power series approximation [21]. Chang and Bentsman extended the continuous time SDRE technique to discrete time under input and state constraints, yielding constrained discrete time SDRE, referred to as CD-SDRE [22]. Babaei and Salamci presented a model reference adaptive control algorithm for a class of nonlinear systems by using a SDRE technique [23]. Nekoo and Geranmehr used a combination of an SDRE controller and SDRE observer to control a class of nonlinear nonaffine control systems [24]. Wang et al. presented a generalized SDRE approach for continuous time nonlinear systems to achieve a mixed nonlinear quadratic regulator and $H^{\infty}$ control performance criteria [25]. Korayem and Nekoo used the finite-time horizon SDRE for controlling a class of nonlinear systems, and the derivations of the SDRE for two classes of systems were presented in the finite-time horizon [26]. Korayem and Nekoo investigated the finite-time optimal and suboptimal controls for timevarying systems with state and control nonlinearities, and a finite-time constraint imposed on the equation leads to a state-dependent differential Riccati equation (SDDRE) [27]. Furthermore, there are some review papers addressing the SDRE method [28-32].

This paper investigated a TSS modeled by a two-piece dumbbell model, which leads to five nonlinear coupled ordinary differential equations. There are two sets of equations of motion formulated. One is based on the first satellite and the other is based on the mass center of the TSS. There are two reasons to formulate the two sets of equations. One is to facilitate their mutual comparison due to the complex formulations. The other is to provide them for different application situations. In order to suppress the librations of the system, the SDRE control is applied, which aims to control a nonlinear system and provides the flexibility of controller

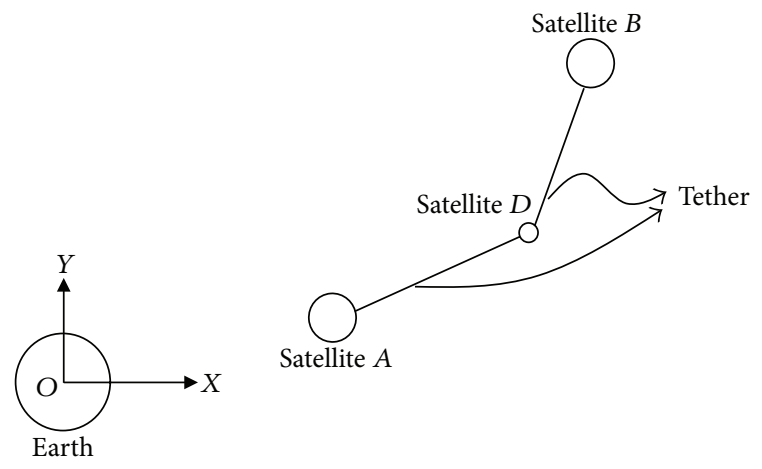

Figure 1: A tethered satellite system.

design. Besides, to increase the convergence rates of the librations, the prescribed degree of stability is incorporated into the SDRE control. This paper is organized as follows. Section 2 formulates the two sets of equations of motion of the TSS. Section 3 presents the SDRE control with the prescribed degree of stability. Section 4 analyzes the nonlinear dynamics of the TSS. Section 5 demonstrates the libration suppressions by the SDRE control. Section 6 summarizes some significant conclusions.

\section{Dynamic Modeling}

A tethered satellite system consists of three satellites connected by a tether, where each satellite is considered as a point mass. Besides, the tether is assumed to be massless, and its length is constant. Thus, the system can be simplified as a two-piece dumbbell model shown in Figure 1, where the three points $A, B$, and $D$ represent three satellites, and they are connected to each other by a constant-length massless tether. These satellites are assumed to be point masses and to be expressed as $m_{A}, m_{B}$, and $m_{D}$, which refer to the points $A, B$, and $D$, respectively. Besides, there is a fixed coordinate system $X-Y$ defined on the center of Earth $O$. In this paper, one neglects the environmental disturbance torques due to the aerodynamics, the solar radiation, the Earth magnetic filed, and so forth.

This section presents two dynamic models to describe the motions of the tethered satellite system. The formulation of the first one is based on satellite $A$, and the other is based on the mass center of the system. Since both formulations describe the same system, they must have the same degrees of freedom, which is equal to five in the system. However, both formulations are expressed in terms of different variables. Each formulation can be used to perform the analysis for a given specific prescribed motion. For instance, if a circular motion is imposed on satellite $A$, the first set of formulations should be adapted. If the same circular motion is imposed on the mass center of the system, the second set of formulations should be selected.

2.1. Formulations Based on Point A. Figure 2(a) shows the coordinate system defined at point $A$, and the position of 


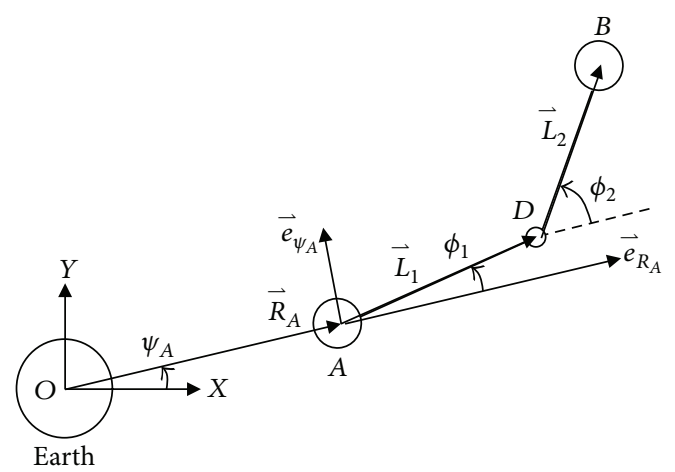

(a)

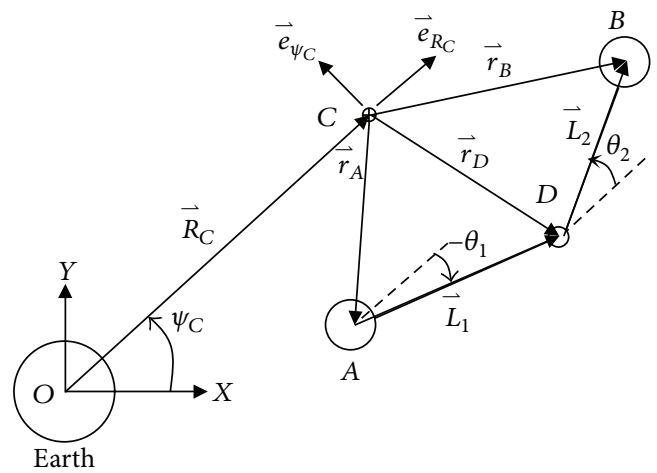

(b)

Figure 2: Simplified tethered satellite systems based on (a) point $A$ and (b) point $C$.

point $A$ is expressed as the orbital radius vector $\vec{R}_{A}$ with the true anomaly $\psi_{A}$. Besides, another coordinate system fixed on point $A$ is defined by the two unit vectors $\vec{e}_{R_{A}}$ and $\vec{e}_{\psi_{A}}$. The relative position vectors from points $A$ to $D$ and from $D$ to $B$ are, respectively, expressed as $\vec{L}_{1}$ and $\vec{L}_{2}$, which can show the configuration of the tether. Referring to Figure 2(a), angles $\phi_{1}$ and $\phi_{2}$, respectively, represent the directions of $\vec{L}_{1}$ and $\vec{L}_{2}$ based on the unit vector $\vec{e}_{R_{A}}$. The two angles are introduced to investigate the libration of the tethered satellite system based on different coordinate systems. If they have an equal value, the system can be treated as a one-piece dumbbell model.

Referring to Figure 2(a), the position vectors of the three point masses from the origin of the $X-Y$ coordinate system are given as

$$
\begin{aligned}
& \vec{R}_{A}=R_{A} \vec{e}_{R_{A}}, \\
& \vec{R}_{B}=\left(R_{A}+L_{1} c_{1}+L_{2} c_{2}\right) \vec{e}_{R_{A}}+\left(L_{1} s_{1}+L_{2} s_{2}\right) \vec{e}_{\psi_{A}}, \\
& \vec{R}_{D}=\left(R_{A}+L_{1} c_{1}\right) \vec{e}_{R_{A}}+L_{1} s_{1} \vec{e}_{\psi_{A}},
\end{aligned}
$$

where $R_{A}, R_{B}, R_{D}, L_{1}$, and $L_{2}$ represent the length of vectors $\vec{R}_{A}, \vec{R}_{B}, \vec{R}_{D}, \vec{L}_{1}$, and $\vec{L}_{2}$, respectively; $c_{1}$ and $c_{2}$ refer to $\cos \phi_{1}$ and $\cos \phi_{2}$, respectively. Note that the three position vectors are expressed in terms of $\vec{e}_{R_{A}}$ and $\vec{e}_{\psi_{A}}$.
By differentiating (1) with respect to time, the velocities of the three vectors are given as

$$
\begin{aligned}
& \dot{\vec{R}}_{A}=\dot{R}_{A} \vec{e}_{R_{A}}+R_{A} \dot{\psi}_{A} \vec{e}_{\psi_{A}}, \\
& \dot{\vec{R}}_{B}=\left[\dot{R}_{A}+\dot{L}_{1}\left(c_{1}-c_{2}\right)-L_{1}\left(\dot{\psi}_{A}+\dot{\phi}_{1}\right) s_{1}\right. \\
& \left.\quad-L_{2}\left(\dot{\psi}_{A}+\dot{\phi}_{2}\right) s_{2}\right] \vec{e}_{R_{A}}+\left[R_{A} \dot{\psi}_{A}+\dot{L}_{1}\left(s_{1}-s_{2}\right)\right. \\
& \left.\quad+L_{1}\left(\dot{\psi}_{A}+\dot{\phi}_{1}\right) c_{1}+L_{2}\left(\dot{\psi}_{A}+\dot{\phi}_{2}\right) c_{2}\right] \vec{e}_{\psi_{A}},
\end{aligned}
$$

$$
\begin{aligned}
& \dot{\vec{R}}_{D}=\left[\dot{R}_{A}+\dot{L}_{1} c_{1}-L_{1}\left(\dot{\psi}_{A}+\dot{\phi}_{1}\right) s_{1}\right] \vec{e}_{R_{A}}+\left[R_{A} \dot{\psi}_{A}\right. \\
& \left.+\dot{L}_{1} s_{1}+L_{1}\left(\dot{\psi}_{A}+\dot{\phi}_{1}\right) c_{1}\right] \vec{e}_{\psi_{A}} .
\end{aligned}
$$

Therefore, the kinetic energy and the potential energy due to the Earth's gravitational field are, respectively, written as

$$
\begin{aligned}
& T=\frac{1}{2} m_{A} \dot{\vec{R}}_{A}^{2}+\frac{1}{2} m_{B} \dot{\vec{R}}_{B}^{2}+\frac{1}{2} m_{D} \dot{\vec{R}}_{D}^{2}, \\
& V=-G M_{e} m_{A} R_{A}^{-1}-G M_{e} m_{B} R_{B}^{-1}-G M_{e} m_{D} R_{D}^{-1},
\end{aligned}
$$

where $G$ and $M_{e}$ are the universal gravitational constant and the Earth mass, respectively.

The nonlinear dynamic equations of motion of the tethered satellite system can be obtained by applying EulerLagrange's equation as

$$
\frac{d}{d t}\left(\frac{\partial L_{g}}{\partial \dot{q}_{i}}\right)-\frac{\partial L_{g}}{\partial q_{i}}=Q_{i}
$$

where $q_{i}$ is the generalized coordinate, $Q_{i}$ is the nonconservative forces corresponding to the generalized coordinate, and $L_{g}$ is the Lagrangian as

$$
L_{g}=T-V
$$

in which $T$ and $V$ are the kinetic energy and the potential energy, respectively. They are expressed in (3) and (4).

Based on the two-piece dumbbell model, the generalized coordinates are $R_{A}, \psi_{A}, \phi_{1}, \phi_{2}$, and $L_{1}$. Besides, the nonconservative forces $Q_{i}$ refer to the driving forces or torques applied to some points and the tether. Substituting (1) to (4) and (6) into (5), one has the nonlinear dynamic equations of motion as follows.

For $q_{i}=R_{A}$, (5) leads to

$$
\begin{aligned}
& \left(m_{A}+m_{B}+m_{D}\right) \ddot{R}_{A} \\
& -\left[\left(m_{B}+m_{D}\right) L_{1} s_{1}+m_{B} L_{2} s_{2}\right] \ddot{\psi}_{A} \\
& -\left[\left(m_{B}+m_{D}\right) L_{1} s_{1}\right] \ddot{\phi}_{1}-\left(m_{B} L_{2} s_{2}\right) \ddot{\phi}_{2}
\end{aligned}
$$




$$
\begin{aligned}
& +\left[\left(m_{B}+m_{D}\right) c_{1}-m_{B} c_{2}\right] \ddot{L}_{1} \\
& -\left(m_{A}+m_{B}+m_{D}\right) R_{A} \dot{\psi}_{A}^{2} \\
& -\left(m_{B}+m_{D}\right)\left[2 \dot{L}_{1}\left(\dot{\psi}_{A}+\dot{\phi}_{1}\right) s_{1}+L_{1}\left(\dot{\psi}_{A}+\dot{\phi}_{1}\right)^{2} c_{1}\right] \\
& +m_{B}\left[2 \dot{L}_{1}\left(\dot{\psi}_{A}+\dot{\phi}_{2}\right) s_{2}-L_{2}\left(\dot{\psi}_{A}+\dot{\phi}_{2}\right)^{2} c_{2}\right] \\
& +G M_{e} m_{A} R_{A}^{-2}+G M_{e} m_{B} R_{B}^{-3} R_{B_{x}}+G M_{e} m_{D} R_{D}^{-3} R_{D_{x}} \\
& =F_{A},
\end{aligned}
$$

where $F_{A}$ is the external force applied to point $A$. One has

$$
\begin{aligned}
& R_{i}=\left(R_{i_{x}}^{2}+R_{i_{y}}^{2}\right)^{1 / 2}, \text { for } i=A, B, D, \\
& {\left[R_{A_{x}}, R_{A_{y}}\right]=\left[R_{A}, 0\right],} \\
& {\left[R_{B_{x}}, R_{B_{y}}\right]} \\
& \quad=\left[R_{A}+L_{1}\left(c_{1}-c_{2}\right)+L c_{2}, L_{1}\left(s_{1}-s_{2}\right)+L s_{2}\right], \\
& {\left[R_{C_{x}}, R_{C_{y}}\right]=\left[R_{A}+L_{1} c_{1}, L_{1} s_{1}\right] .}
\end{aligned}
$$

Note that (9) to (11) are expressed based on the coordinate system, $\vec{e}_{R_{A}}$ and $\vec{e}_{\psi_{A}}$.

For $q_{i}=\psi_{A}$, (5) leads to

$$
\begin{aligned}
& {\left[-\left(m_{B}+m_{D}\right) L_{1} s_{1}-m_{B} L_{2} s_{2}\right] \ddot{R}_{A}} \\
& +\left[\left(m_{A}+m_{B}+m_{D}\right) R_{A}^{2}\right. \\
& +\left(m_{B}+m_{D}\right)\left(L_{1}^{2}+2 R_{A} L_{1} c_{1}\right) \\
& \left.+m_{B}\left(L_{2}^{2}+2 L_{1} L_{2} c_{12}+2 R_{A} L_{2} c_{2}\right)\right] \ddot{\psi}_{A} \\
& +\left[\left(m_{B}+m_{D}\right)\left(L_{1}^{2}+R_{A} L_{1} c_{1}\right)+m_{B} L_{1} L_{2} c_{12}\right] \ddot{\phi}_{1} \\
& +m_{B}\left(L_{2}^{2}+L_{1} L_{2} c_{12}+R_{A} L_{2} c_{2}\right) \ddot{\phi}_{2} \\
& +\left[\left(m_{B}+m_{D}\right) R_{A} s_{1}+m_{B}\left(-R_{A} s_{2}+L s_{12}\right)\right] \ddot{L}_{1} \\
& +2\left(m_{A}+m_{B}+m_{D}\right) R_{A} \dot{R}_{A} \dot{\psi}_{A}+\left(m_{B}+m_{D}\right) \\
& \text {. }\left[-R_{A} L_{1}\left(2 \dot{\psi}_{A}+\dot{\phi}_{1}\right) \dot{\phi}_{1} s_{1}+2 L_{1} \dot{L}_{1}\left(\dot{\psi}_{A}+\dot{\phi}_{1}\right)\right. \\
& \left.+2 R_{A} \dot{L}_{1}\left(\dot{\psi}_{A}+\dot{\phi}_{1}\right) c_{1}+2 L_{1} \dot{R}_{A} \dot{\psi}_{A} c_{1}\right] \\
& +m_{B}\left[-2 L_{2} \dot{L}_{1}\left(\dot{\psi}_{A}+\dot{\phi}_{2}\right)\right. \\
& +\left(L_{2}-L_{1}\right) \dot{L}_{1}\left(2 \dot{\psi}_{A}+\dot{\phi}_{1}+\dot{\phi}_{2}\right) c_{12} \\
& -L L_{2}\left(2 \dot{\psi}_{A}+\dot{\phi}_{1}+\dot{\phi}_{2}\right)\left(\dot{\phi}_{1}-\dot{\phi}_{2}\right) s_{12} \\
& +L \dot{L}_{1}\left(\dot{\phi}_{1}-\dot{\phi}_{2}\right) c_{12}+2 L_{2} \dot{R}_{A} \dot{\psi}_{A} c_{2} \\
& \left.-2 R_{A} \dot{L}_{1}\left(\dot{\psi}_{A}+\dot{\phi}_{2}\right) c_{2}-R_{A} L_{2}\left(2 \dot{\psi}_{A}+\dot{\phi}_{2}\right) \dot{\phi}_{2} s_{2}\right] \\
& =T_{O} \text {, }
\end{aligned}
$$

where $T_{O}$ is the external torque applied to point $O$ and $c_{12}$ and $s_{12}$ refer to $\cos \left(\phi_{1}-\phi_{2}\right)$ and $\sin \left(\phi_{1}-\phi_{2}\right)$, respectively.

For $q_{i}=\phi_{1}$, (5) leads to

$$
\begin{aligned}
& -\left(m_{B}+m_{D}\right) L_{1} s_{1} \ddot{R}_{A}+\left[\left(m_{B}+m_{D}\right)\left(L_{1}^{2}+L_{1} R_{A} c_{1}\right)\right. \\
& \left.+m_{B} L_{1} L_{2} c_{12}\right] \ddot{\psi}_{A}+\left(m_{B}+m_{D}\right) L_{1}^{2} \ddot{\phi}_{1} \\
& +m_{B} L_{1} L_{2} c_{12} \ddot{\phi}_{2}+m_{B} L_{1} s_{12} \ddot{L}_{1}+\left(m_{B}+m_{D}\right) \\
& \quad+\left[2 L_{1} \dot{L}_{1}\left(\dot{\psi}_{A}+\dot{\phi}_{1}\right)+R_{A} L_{1} s_{1} \dot{\psi}_{A}^{2}+2 L_{1} \dot{R}_{A} \dot{\psi}_{A} c_{1}\right] \\
& +m_{B}\left[-2 L_{1} \dot{L}_{1}\left(\dot{\psi}_{A}+\dot{\theta}_{2}\right) c_{12}\right. \\
& \left.\quad+L_{1} L_{2}\left(\dot{\psi}_{A}+\dot{\theta}_{2}\right)^{2} s_{12}\right]+G M_{e} m_{B} R_{B}^{-3}\left(-R_{B_{x}} L_{1} s_{1}\right. \\
& \left.+R_{B_{y}} L_{1} c_{1}\right)+G M_{e} m_{D} R_{D}^{-3}\left(-R_{D_{x}} L_{1} s_{1}+R_{D_{y}} L_{1} c_{1}\right) \\
& \quad=T_{A},
\end{aligned}
$$

where $T_{A}$ is the external torque applied to point $A$.

For $q_{i}=\phi_{2}$, (5) leads to

$$
\begin{aligned}
& -\left(m_{B} L_{2} s_{2}\right) \ddot{R}_{A}+m_{B}\left(L_{2}^{2}+L_{1} L_{2} c_{12}+R_{A} L_{2} c_{2}\right) \ddot{\psi}_{A} \\
& +\left(m_{B} L_{1} L_{2} c_{12}\right) \ddot{\phi}_{1}+\left(m_{B} L_{2}^{2}\right) \ddot{\phi}_{2}+\left(m_{2} L_{2} s_{12}\right) \ddot{L}_{1} \\
& +m_{B}\left[R_{A} L_{2} s_{2} \dot{\psi}_{A}^{2}-2 L_{2} \dot{L}_{1}\left(\dot{\psi}_{A}+\dot{\phi}_{2}\right)\right. \\
& +2 L_{2} \dot{R}_{A} \dot{\psi}_{A} c_{2}+2 L_{2} c_{12} \dot{L}_{1}\left(\dot{\psi}_{A}+\dot{\phi}_{1}\right) \\
& \left.-L_{1} L_{2}\left(\dot{\psi}_{A}+\dot{\phi}_{1}\right)^{2} s_{12}\right]+G M_{e} m_{B} R_{B}^{-3}\left(-R_{B_{x}} L_{2} s_{2}\right. \\
& \left.+R_{B_{y}} L_{2} c_{2}\right)=T_{D},
\end{aligned}
$$

where $T_{D}$ is the external torque applied to point $D$.

For $q_{i}=L_{1}$, (5) leads to

$$
\begin{aligned}
& {\left[\left(m_{B}+m_{D}\right) c_{1}-m_{B} c_{2}\right] \ddot{R}_{A}+\left[\left(m_{B}+m_{D}\right) R_{A} s_{1}\right.} \\
& \left.\quad+m_{B}\left(-R_{A} s_{2}+L_{1} s_{12}\right)\right] \ddot{\psi}_{A}+m_{B} L_{1} s_{12} \ddot{\phi}_{1} \\
& +m_{B} L_{2} s_{12} \ddot{\phi}_{2}+\left[\left(m_{B}+m_{D}\right)+m_{B}\left(1-2 c_{12}\right)\right] \ddot{L}_{1} \\
& +\left(m_{B}+m_{D}\right)\left[2 \dot{R}_{A} \dot{\psi}_{A} s_{1}-R_{A} \dot{\psi}_{A}^{2} c_{1}\right. \\
& \left.\quad-L_{1}\left(\dot{\psi}_{A}+\dot{\phi}_{1}\right)^{2}\right]+m_{B}\left[-2 \dot{R}_{A} \dot{\psi}_{A} s_{2}+R_{A} \dot{\psi}_{A}^{2} c_{2}\right. \\
& +L_{2}\left(\dot{\psi}_{A}+\dot{\phi}_{2}\right)^{2}+L_{1}\left(\dot{\psi}_{A}+\dot{\phi}_{1}\right)^{2} c_{12} \\
& \left.\quad-L_{2}\left(\dot{\psi}_{A}+\dot{\phi}_{2}\right)^{2} c_{12}+2 \dot{L}_{1}\left(\dot{\phi}_{1}-\dot{\phi}_{2}\right) s_{12}\right] \\
& +G M_{e} m_{B} R_{B}^{-3}\left[R_{B_{x}}\left(c_{1}-c_{2}\right)+R_{B_{y}}\left(s_{1}-s_{2}\right)\right] \\
& +G M_{e} m_{D} R_{D}^{-3}\left(R_{D_{x}} c_{1}+R_{D_{y}} s_{1}\right)=F_{L},
\end{aligned}
$$

where $F_{L}$ is the external force applied to the tether. 
Equations (7) and (12) to (15) are the nonlinear dynamic equations of motion for the tethered satellite system based on the coordinate system $\vec{e}_{R_{A}}$ and $\vec{e}_{\psi_{A}}$.

2.2. Formulations Based on the Mass Center. Figure 2(b) shows the coordinate system defined at the mass center, where point $C$ represents the mass center of the tethered satellite system, and the position vector from points $O$ to $C$ is expressed as $\vec{R}_{C}$ with the true anomaly $\psi_{C}$. The relative position vectors from points $C$ to the three point masses are $\vec{r}_{A}, \vec{r}_{B}$, and $\vec{r}_{D}$. Referring to Figure 2(b), another coordinate system fixed on point $C$ is defined by the two unit vectors $\vec{e}_{R_{C}}$ and $\vec{e}_{\psi_{C}}$. Referring to Figure 2(b), angles $\theta_{1}$ and $\theta_{2}$, respectively, represent the directions of $\vec{L}_{1}$ and $\vec{L}_{2}$ based on the unit vector $\vec{e}_{R_{\mathrm{C}}}$. Since the total length of the tether is a constant value, it can be written as

$$
L=L_{1}+L_{2}
$$

where $L_{1}$ and $L_{2}$ are the lengths of vectors $\vec{L}_{1}$ and $\vec{L}_{2}$, respectively.

To formulate the dynamic equations of the two-piece dumbbell model, one will apply Euler-Lagrange's equation, and the position and velocity of each point mass will be expressed in terms of the relative vectors $\vec{L}_{1}$ and $\vec{L}_{2}$. Furthermore, they will be written based on the coordinate system $\left(\vec{e}_{R_{C}}, \vec{e}_{\psi_{C}}\right)$.

Referring to Figure 2(b), one has these two equations as

$$
\begin{aligned}
-\vec{r}_{A}+\vec{r}_{D} & =\vec{L}_{1}, \\
\vec{r}_{B}-\vec{r}_{D} & =\vec{L}_{2} .
\end{aligned}
$$

Besides, since point $C$ is the mass center of the tethered satellite system, one has the equation as

$$
\rho_{A} \vec{r}_{A}+\rho_{B} \vec{r}_{B}+\rho_{D} \vec{r}_{D}=0,
$$

where the mass ratios are written as

$$
\begin{aligned}
& \rho_{A}=\frac{m_{A}}{M}, \\
& \rho_{B}=\frac{m_{B}}{M}, \\
& \rho_{D}=\frac{m_{D}}{M}
\end{aligned}
$$

in which the total mass of the system is given by

$$
M=m_{A}+m_{B}+m_{D}
$$

Simultaneously solving (17) and (18) leads to

$$
\begin{aligned}
& \vec{r}_{A}=-\left(1-\rho_{A}\right) \vec{L}_{1}-\rho_{B} \vec{L}_{2}, \\
& \vec{r}_{B}=\rho_{A} \vec{L}_{1}+\left(1-\rho_{B}\right) \vec{L}_{2}, \\
& \vec{r}_{D}=\rho_{A} \vec{L}_{1}-\rho_{B} \vec{L}_{2} .
\end{aligned}
$$

Note that (21) expresses the relative vectors $\vec{r}_{A}, \vec{r}_{B}$, and $\vec{r}_{D}$ in terms of the relative vectors $\vec{L}_{1}$ and $\vec{L}_{2}$.

By taking the time derivatives of (21), the velocities of the relative vectors $\vec{r}_{A}, \vec{r}_{B}$, and $\vec{r}_{D}$ are given as

$$
\begin{aligned}
& \dot{\vec{r}}_{A}=-\left(1-\rho_{A}\right) \dot{\vec{L}}_{1}-\rho_{B} \dot{\vec{L}}_{2}, \\
& \dot{\vec{r}}_{B}=\rho_{A} \dot{\vec{L}}_{1}+\left(1-\rho_{B}\right) \dot{\vec{L}}_{2}, \\
& \dot{\vec{r}}_{A}=\rho_{A} \dot{\vec{L}}_{1}-\rho_{B} \dot{\vec{L}}_{2} .
\end{aligned}
$$

Referring to Figure 2(b) again, the relative vectors $\vec{L}_{1}$ and $\vec{L}_{2}$ can be expressed in the coordinate system $\left(\vec{e}_{r}, \vec{e}_{\psi}\right)$ as

$$
\begin{aligned}
& \vec{L}_{1}=L_{1} c_{1} \vec{e}_{R_{C}}+L_{1} s_{1} \vec{e}_{\psi_{C}}, \\
& \vec{L}_{2}=L_{2} c_{2} \vec{e}_{R_{C}}+L_{2} s_{2} \vec{e}_{\psi_{C}},
\end{aligned}
$$

where $c_{1}, c_{2}, s_{1}$, and $s_{2}$ refer to $\cos \theta_{1}, \cos \theta_{2}, \sin \theta_{1}$, and $\sin \theta_{2}$, respectively.

By taking the time derivatives of (23), the velocity of the relative vectors $\vec{L}_{1}$ and $\vec{L}_{2}$ is expressed as

$$
\begin{aligned}
\dot{\vec{L}}_{1}= & \left(\dot{L}_{1} c_{1}-L_{1} \dot{\theta}_{1} s_{1}-L_{1} \dot{\psi}_{C} s_{1}\right) \vec{e}_{R_{C}} \\
& +\left(\dot{L}_{1} s_{1}+L_{1} \dot{\theta}_{1} c_{1}+L_{1} \dot{\psi}_{C} c_{1}\right) \vec{e}_{\psi_{C}}, \\
\dot{\vec{L}}_{2}= & \left(\dot{L}_{2} c_{2}-L_{2} \dot{\theta}_{2} s_{s}-L_{2} \dot{\psi}_{C} s_{2}\right) \vec{e}_{R_{C}} \\
& +\left(\dot{L}_{2} s_{2}+L_{2} \dot{\theta}_{2} c_{2}+L_{2} \dot{\psi}_{C} c_{2}\right) \vec{e}_{\psi_{C}} .
\end{aligned}
$$

To obtain the dynamic equations of the system by applying Euler-Lagrange's equation, the kinetic energy is written as

$$
\begin{aligned}
T= & \frac{1}{2} m_{A}\left(\dot{\vec{R}}_{C}+\dot{\vec{r}}_{A}\right)^{2}+\frac{1}{2} m_{B}\left(\dot{\vec{R}}_{C}+\dot{\vec{r}}_{B}\right)^{2} \\
& +\frac{1}{2} m_{D}\left(\dot{\vec{R}}_{C}+\dot{\vec{r}}_{D}\right)^{2} \\
= & \frac{1}{2} M \dot{\vec{R}}_{C}^{2}+\frac{1}{2} m_{A} \dot{\vec{r}}_{A}^{2}+\frac{1}{2} m_{B} \dot{\vec{r}}_{B}^{2}+\frac{1}{2} m_{D} \dot{\vec{r}}_{D}^{2} \\
= & \frac{1}{2} M \dot{\vec{R}}_{C}^{2}+\frac{1}{2} a_{1} \dot{\vec{L}}_{1}^{2}+\frac{1}{2} a_{2} \dot{\vec{L}}_{2}^{2}+a_{3} \dot{\vec{L}}_{1} \cdot \dot{\vec{L}}_{2},
\end{aligned}
$$

where

$$
\begin{aligned}
& a_{1}=m_{A}\left(1-\rho_{A}\right)^{2}+m_{B} \rho_{A}^{2}+m_{D} \rho_{A}^{2}, \\
& a_{2}=m_{A} \rho_{B}^{2}+m_{B}\left(1-\rho_{B}\right)^{2}+m_{D} \rho_{B}^{2}, \\
& a_{3}=m_{A}\left(1-\rho_{A}\right) \rho_{B}+m_{B} \rho_{A}\left(1-\rho_{B}\right)-m_{D} \rho_{A} \rho_{B} .
\end{aligned}
$$

And the potential energy is written as

$$
\begin{aligned}
V= & -G M_{e} m_{A}\left|\vec{R}_{C}+\vec{r}_{A}\right|^{-1}-G M_{e} m_{B}\left|\vec{R}_{C}+\vec{r}_{B}\right|^{-1} \\
& -G M_{e} m_{D}\left|\vec{R}_{C}+\vec{r}_{D}\right|^{-1} .
\end{aligned}
$$


Using Euler-Lagrange's equation shown in (5), one obtains the equations of motion as follows.

For $q_{i}=R_{C}$, (5) leads to

$$
\begin{aligned}
M \ddot{R}_{C} & -M R_{C} \dot{\psi}_{C}^{2}+G M_{e} m_{A} R_{A}^{-3} R_{A_{x}}+G M_{e} m_{B} R_{B}^{-3} R_{B_{x}} \\
& +G M_{e} m_{D} R_{D}^{-3} R_{D_{x}}=F_{C},
\end{aligned}
$$

where $F_{C}$ is the external force applied to the center of the system and

$$
\begin{aligned}
& R_{i}=\left(R_{i_{x}}^{2}+R_{i_{y}}^{2}\right)^{1 / 2}, \text { for } i=A, B, D, \\
& \quad\left[R_{A_{x}}, R_{A_{y}}\right]=\left[R_{C}-\left(1-\rho_{A}\right) L_{1} c_{1}-\rho_{B} L_{2} c_{2},\right. \\
& \left.\quad-\left(1-\rho_{A}\right) L_{1} s_{1}-\rho_{B} L_{2} s_{2}\right], \\
& \quad\left[R_{B_{x}}, R_{B_{y}}\right]=\left[R_{C}+\rho_{A} L_{1} c_{1}+\left(1-\rho_{B}\right) L_{2} c_{2}, \rho_{A} L_{1} s_{1}\right. \\
& \left.\quad+\left(1-\rho_{B}\right) L_{2} s_{2}\right], \\
& \quad\left[R_{C_{x}}, R_{C_{y}}\right]=\left[R_{C}+\rho_{A} L_{1} c_{1}-\rho_{B} L_{2} c_{2}, \rho_{A} L_{1} s_{1}\right. \\
& \left.\quad-\rho_{B} L_{2} s_{2}\right] .
\end{aligned}
$$$$
\text { For } q_{i}=\psi_{C} \text {, (5) leads to }
$$

$$
\begin{aligned}
\left(M R_{C}^{2}\right. & \left.+a_{1} L_{1}^{2}+a_{2} L_{2}^{2}+2 a_{3} L_{1} L_{2} c_{12}\right) \ddot{\psi}_{C} \\
& +\left(a_{1} L_{1}^{2}+a_{3} L_{1} L_{2} c_{12}\right) \ddot{\theta}_{1} \\
& +\left(a_{2} L_{2}^{2}+a_{3} L_{1} L_{2} c_{12}\right) \ddot{\theta}_{2}+\left(a_{3} L s_{12}\right) \ddot{L}_{1} \\
& +2 M R_{C} \dot{R}_{C} \dot{\psi}_{C}+2 a_{1} L_{1} \dot{L}_{1}\left(\dot{\psi}_{C}+\dot{\theta}_{1}\right) \\
& -2 a_{2} L_{2} \dot{L}_{1}\left(\dot{\psi}_{C}+\dot{\theta}_{2}\right) \\
& +a_{3}\left(L_{2}-L_{1}\right) c_{12} \dot{L}_{1}\left(2 \dot{\psi}_{C}+\dot{\theta}_{1}+\dot{\theta}_{2}\right) \\
& -a_{3} L_{1} L_{2} s_{12}\left(2 \dot{\psi}_{C}+\dot{\theta}_{1}+\dot{\theta}_{2}\right)\left(\dot{\theta}_{1}-\dot{\theta}_{2}\right) \\
& +a_{3} L \dot{L}_{1} c_{12}\left(\dot{\theta}_{1}-\dot{\theta}_{2}\right)=T_{O},
\end{aligned}
$$

where $T_{O}$ is the external torque at point $O, c_{12}=\cos \left(\theta_{1}-\theta_{2}\right)$, and $s_{12}=\sin \left(\theta_{1}-\theta_{2}\right)$.

For $q_{i}=\theta_{1}$, (5) leads to

$$
\begin{aligned}
\left(a_{1} L_{1}^{2}\right. & \left.+a_{3} L_{1} L_{2} c_{12}\right) \ddot{\psi}_{C}+\left(a_{1} L_{1}^{2}\right) \ddot{\theta}_{1}+\left(a_{3} L_{1} L_{2} c_{12}\right) \ddot{\theta}_{2} \\
& +\left(a_{3} L_{1} s_{12}\right) \ddot{L}_{1}+2 a_{1} L_{1} \dot{L}_{1}\left(\dot{\psi}_{C}+\dot{\theta}_{1}\right) \\
& +a_{3} L_{1} L_{2} c_{12}\left(\dot{\psi}_{C}+\dot{\theta}_{2}\right)^{2} s_{12} \\
& -2 a_{3} L_{1} \dot{L}_{1}\left(\dot{\psi}_{C}+\dot{\theta}_{2}\right) c_{12} \\
& +G M_{e} m_{A} R_{A}^{-3}\left(1-\rho_{A}\right) L_{1}\left(s_{1} R_{A_{x}}-c_{1} R_{A_{y}}\right) \\
& +G M_{e} m_{B} R_{B}^{-3} \rho_{A} L_{1}\left(-s_{1} R_{B_{x}}+c_{1} R_{B_{y}}\right) \\
& +G M_{e} m_{D} R_{D}^{-3} \rho_{A} L_{1}\left(-s_{1} R_{D_{x}}+c_{1} R_{D_{y}}\right)=T_{A}
\end{aligned}
$$

where $T_{A}$ is the external torque applied at point $A$.
For $q_{i}=\theta_{2},(5)$ leads to

$$
\begin{aligned}
\left(a_{2} L_{2}^{2}+a_{3} L_{1} L_{2} c_{12}\right) \ddot{\psi}_{C}+\left(a_{3} L_{1} L_{2} c_{12}\right) \ddot{\theta}_{1}+\left(a_{2} L_{2}^{2}\right) \ddot{\theta}_{2} \\
+\left(a_{3} L_{2} s_{12}\right) \ddot{L}_{1}-2 a_{2} L_{2} \dot{L}_{1}\left(\dot{\psi}_{C}+\dot{\theta}_{2}\right) \\
\quad-a_{3} L_{1} L_{2} c_{12}\left(\dot{\psi}_{C}+\dot{\theta}_{1}\right)^{2} s_{12} \\
+2 a_{3} L_{2} \dot{L}_{1}\left(\dot{\psi}_{C}+\dot{\theta}_{1}\right) c_{12} \\
+G M_{e} m_{A} R_{A}^{-3} \rho_{B} L_{2}\left(s_{2} R_{A_{x}}-c_{2} R_{A_{y}}\right) \\
+G M_{e} m_{B} R_{B}^{-3}\left(1-\rho_{B}\right) L_{2}\left(-s_{2} R_{B_{x}}+c_{2} R_{B_{y}}\right) \\
+G M_{e} m_{D} R_{D}^{-3} \rho_{B} L_{2}\left(s_{2} R_{D_{x}}-c_{2} R_{D_{y}}\right)=T_{D}
\end{aligned}
$$

where $T_{D}$ is the external torque applied at point $D$.

For $q_{i}=L_{1}$, (5) leads to

$$
\begin{aligned}
& \left(a_{3} L s_{12}\right) \ddot{\psi}_{C}+\left(a_{3} L_{1} s_{12}\right) \ddot{\theta}_{1}+\left(a_{3} L_{2} s_{12}\right) \ddot{\theta}_{2}+\left(a_{1}+a_{2}\right. \\
& \left.-2 a_{3} c_{12}\right) \ddot{L}_{1}-a_{1} L_{1}\left(\dot{\psi}_{C}+\dot{\theta}_{1}\right)^{2}+a_{2} L_{2}\left(\dot{\psi}_{C}+\dot{\theta}_{2}\right)^{2} \\
& -a_{3}\left(L_{2}-L_{1}\right)\left(\dot{\psi}_{C}+\dot{\theta}_{1}\right)\left(\dot{\psi}_{C}+\dot{\theta}_{2}\right) c_{12} \\
& +2 a_{3} s_{12} \dot{L}_{1}\left(\dot{\theta}_{1}-\dot{\theta}_{2}\right)+a_{3} L_{1} c_{12}\left(\dot{\psi}_{C}+\dot{\theta}_{1}\right)\left(\dot{\theta}_{1}\right. \\
& \left.-\dot{\theta}_{2}\right)+a_{3} L_{2} c_{12}\left(\dot{\psi}_{C}+\dot{\theta}_{2}\right)\left(\dot{\theta}_{1}-\dot{\theta}_{2}\right) \\
& +G M_{e} m_{A} R_{A}^{-3}\left\{\left[-\left(1-\rho_{A}\right) c_{1}+\rho_{B} c_{2}\right] R_{A_{x}}\right. \\
& \left.+\left[-\left(1-\rho_{A}\right) s_{1}+\rho_{B} s_{2}\right] R_{A_{y}}\right\} \\
& +G M_{e} m_{B} R_{B}^{-3}\left\{\left[\rho_{A} c_{1}-\left(1-\rho_{B}\right) c_{2}\right] R_{B_{x}}\right. \\
& \left.+\left[\rho_{A} s_{1}-\left(1-\rho_{B}\right) s_{2}\right] R_{B_{y}}\right\} \\
& +G M_{e} m_{D} R_{D}^{-3}\left\{\left[\rho_{A} c_{1}+\rho_{B} c_{2}\right] R_{D_{x}}\right. \\
& \left.+\left[\rho_{A} s_{1}+\rho_{B} s_{2}\right] R_{D_{y}}\right\}=F_{L},
\end{aligned}
$$

where $F_{L}$ is the external force applied at the tether.

Equations (28) and (30) to (33) are the nonlinear dynamic equations of motion for the tethered satellite system based on the coordinate system $\left(\vec{e}_{R_{C}}, \vec{e}_{\psi_{C}}\right)$. Note that the term related to $\ddot{R}_{C}$ only appears in (28) but not in (30) to (33). This property is not shown in the equations of motion based on point $A$.

\section{SDRE Control with a Specified Degree of Stability}

A nonlinear and observable system is written as

$$
\dot{x}(t)=f(x)+g(x) u(t), \quad x(0)=x_{0},
$$


where $x(t)$ and $u(t)$ are the state and input vectors, respectively.

To perform a regulation problem, an infinite-time performance index is written as

$$
\begin{aligned}
J( & \left.x_{0}, u(t)\right) \\
& =\frac{1}{2} \int_{0}^{\infty}\left[x^{T}(x) Q(x) x(t)+u^{T}(t) R(x) u(t)\right] d t,
\end{aligned}
$$

where $Q(t) \geq 0$ and $R(t)>0$ are state-dependent weighting matrices.

To apply the LQR, (34) can be factorized in a linear-like structure as

$$
\dot{x}(t)=A(x) x(t)+B(x) u(t),
$$

where $f(x)=A(x) x(t)$ and $g(x)=B(x)$. There is an infinite number of forms to factorize $f(x)$ as $A(x) x(t)$, so this property increases the flexibility of the SDRE.

Solving (35) and (36) by following the formulation of the LQR leads to

$$
u(t)=-K(x) x(t)=-R^{-1}(x) B^{T}(x) P(x) x(t),
$$

where $P(x)$ is the symmetric and positive-definite solution of the algebraic state-dependent Riccati equation as

$$
\begin{aligned}
P(x) & A(x)+A^{T}(x) P(x) \\
& -P(x) B(x) R^{-1}(x) B^{T}(x) P(x)+Q(x)=0 .
\end{aligned}
$$

To ensure the closed-loop system with a specified degree of stability, the performance index is rewritten as

$$
\begin{aligned}
& J\left(x_{0}, u(t)\right)=\frac{1}{2} \int_{0}^{\infty} e^{2 \alpha t}\left[x^{T}(x) Q(x) x(t)\right. \\
& \left.+u^{T}(t) R(x) u(t)\right] d t,
\end{aligned}
$$

where $\alpha$ is a positive parameter. Note that the pair $[A+\alpha I, B]$ is completely stabilizable, where $I$ is an identity matrix.

Define new state variables and controls as

$$
\begin{aligned}
& \widehat{x}(t)=e^{\alpha t} x(t), \\
& \widehat{u}(t)=e^{\alpha t} u(t) .
\end{aligned}
$$

Using (40), the system equations shown in (36) can be modified as

$$
\dot{\hat{x}}(t)=(A(x)+\alpha I) \widehat{x}(t)+B \widehat{u}(t) .
$$

Note that the initial condition is the same as $\widehat{x}(0)=x(0)=x_{0}$. Also, the performance index is transformed as

$$
\begin{aligned}
J( & \left.x_{0}, \widehat{u}(t)\right) \\
& =\frac{1}{2} \int_{0}^{\infty}\left[\widehat{x}^{T}(x) Q(x) \widehat{x}(t)+\widehat{u}^{T}(t) R(x) \widehat{u}(t)\right] d t .
\end{aligned}
$$

Solving (41) and (42) by following the formulation of the LQR leads to

$$
\widehat{u}(t)=-\bar{K}(x) \widehat{x}(t)=-R^{-1}(x) B^{T}(x) \bar{P}(x) \widehat{x}(t),
$$

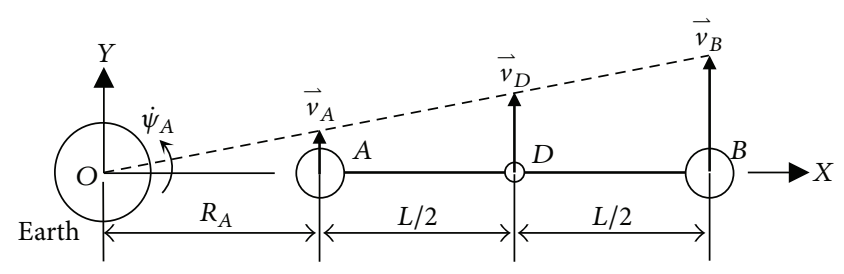

FIgURE 3: Initial configuration for free libration.

where $\bar{P}(x)$ is the symmetric and positive-definite solution of the algebraic state-dependent Riccati equation as

$$
\begin{aligned}
\bar{P}(x) & {[A(x)+\alpha I]+[A(x)+\alpha I]^{T} \bar{P}(x) } \\
- & \bar{P}(x) B(x) R^{-1}(x) B^{T}(x) \bar{P}(x)+Q(x)=0 .
\end{aligned}
$$

Substituting (43) into (41) leads to the optimal closedloop system as

$$
\begin{aligned}
\dot{\hat{x}}( & t) \\
\quad= & {\left[A(x)+\alpha I-B(x) R^{-1}(x) B^{T}(x) \bar{P}(x)\right] \widehat{x}(t) . }
\end{aligned}
$$

Roughly speaking, the system seemingly has eigenvalues with real parts less than $-\alpha$, so the system is treated with a degree of stability of at least $\alpha$.

Based on (43), the optimal control can be transformed to

$$
\begin{aligned}
u(t) & =e^{-\alpha t} \widehat{u}(t)=-\bar{K}(x) x \\
& =-R^{-1}(x) B^{T}(x) \bar{P}(x) x(t) .
\end{aligned}
$$

Note that the original control (see (37)) has the same structure as the optimal control (see (46)) of the modified system.

\section{Nonlinear Dynamic Analysis}

This section presents the nonlinear dynamic analysis without applying any control theory.

4.1. Free Libration. To demonstrate the consistence of the proposed formulations, the numerical simulations of free libration are performed. The initial configuration is that three satellites and the mass center of the Earth are collinear, and satellite $D$ is at the middle point between satellites $A$ and $B$. Figure 3 shows the initial configuration, where the distance from the Earth center to satellite $A$ is $42,522 \mathrm{~km}$ and the length of the tether is $10,000 \mathrm{~km}$. The three satellites have the same initial orbital rate $\dot{\psi}_{A}=7.2923 \times 10^{-5} \mathrm{rad} / \mathrm{s}$. Hence, the initial velocities of these satellites can be determined and are expressed as $\vec{v}_{A}, \vec{v}_{B}$, and $\vec{v}_{D}$ shown in Figure 3 . The masses of satellites are identical as $10,000 \mathrm{~kg}$, and the mass of satellite is $100 \mathrm{~kg}$. Based on the aforementioned initial state, the initial conditions are given as $R_{A}=42,522 \mathrm{~km}, \psi_{A}=0, \phi_{1}=0$, $\phi_{2}=0, L_{1}=5,000 \mathrm{~km}, \dot{R}_{A}=0, \dot{\psi}_{A}=7.2923 \times 10^{-5} \mathrm{rad} / \mathrm{s}$, $\dot{\phi}_{1}=0, \dot{\phi}_{2}=0$, and $\dot{L}_{1}=0$ for the formulations based on point $A$. The initial conditions are $R_{C}=47,522 \mathrm{~km}, \psi_{C}=0, \theta_{1}=0$, $\theta_{2}=0, L_{1}=5000 \mathrm{~km}, \dot{R}_{C}=0, \dot{\psi}_{C}=7.2923 \times 10^{-5} \mathrm{rad} / \mathrm{s}$, 


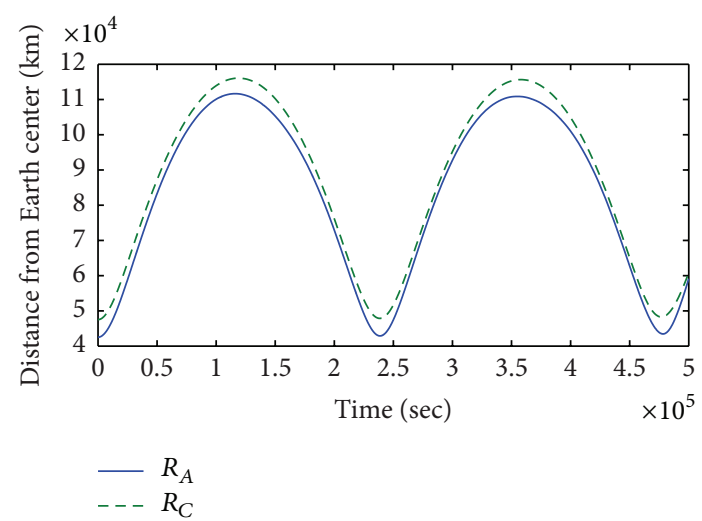

FIgURE 4: The distances from Earth center as functions of time for free libration.

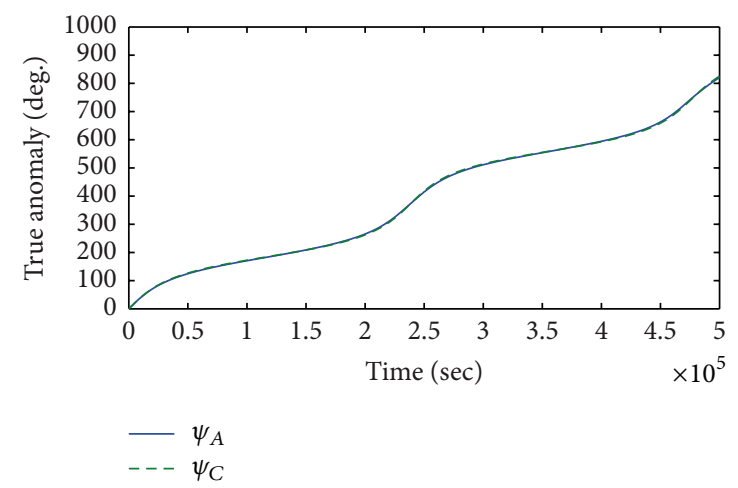

FIgURE 5: True anomaly as functions of time for free libration. $\dot{\theta}_{1}=0, \dot{\theta}_{2}=0$, and $\dot{L}_{1}=0$ for the formulations based on point $C$.

Since both formulations represent the same system and the same initial state is applied, the numerical simulations must be identical. Figure 4 shows distances $R_{A}$ and $R_{C}$ as functions to time. The results show that the distances periodically change and one period is around $2.389 \times 10^{4}$ seconds. Besides, the mean values of $R_{A}$ and $R_{C}$ are $7.7083 \times$ $10^{4}$ and $8.1791 \times 10^{4} \mathrm{~km}$, respectively. Also, their amplitude is $3.4561 \times 10^{4}$ and $3.4269 \times 10^{4} \mathrm{~km}$, respectively. Figure 5 shows the true anomalies $\psi_{A}$ and $\psi_{C}$ as functions of time. The results show that they do not linearly change with time. Figure 6 shows the libration angles $\phi_{1}$ and $\phi_{2}$ and the tether length $L_{1}$ as functions of time. Since the total length of the tether is $10,000 \mathrm{~km}$, the tether length $L_{1}$ must be less than the total length. This figure shows that length $L_{1}$ changes between 0 and $10,000 \mathrm{~km}$. Besides, while length $L_{1}$ reaches maximum value $10,000 \mathrm{~km}$, the libration angle $\phi_{2}$ will have the change of $360^{\circ}$. Similarly, while length $L_{1}$ reaches the minimum value 0 , the libration angle $\phi_{1}$ also has the change of $360^{\circ}$. Figure 7 shows the libration angles $\theta_{1}$ and $\theta_{2}$ and tether length $L_{1}$ as functions of time, and this figure is similar to Figure 6.

4.2. Free Librations with a Prescribed Function $L_{1}(t)$. One considers that satellite $D$ is a climber, which will move along the tether based on a prescribed function. The climber is initially located at $L_{10}=0.1 \mathrm{~km}$. It ascends while accelerating until reaching $L_{11}=0.1 L$. It cruises at a constant velocity $v_{c}$ until $L_{12}=L_{1 f}-0.1 L$, where $L_{1 f}$ is the final location of the climber. During the acceleration and deceleration periods, the velocity varies as a sinusoidal function. Hence, the motion of the climber is described by the following piecewise function as

$$
L_{1}(t)= \begin{cases}L_{10}+\frac{v_{c}}{2}\left(t-\frac{t_{1}}{\pi} \sin \frac{\pi t}{t_{1}}\right), & \text { for } 0 \leq t \leq t_{1} \\ L_{10}+v_{c}\left(t-\frac{t_{1}}{2}\right), & \text { for } t_{1}<t \leq t_{2} \\ L_{10}+\frac{v_{c}}{2}\left(t+t_{2}-t_{1}+\frac{t_{f}-t_{2}}{\pi} \sin \frac{\pi\left(t-t_{2}\right)}{t_{f}-t_{2}}\right), & \text { for } t_{2}<t \leq t_{f} \\ L_{1 f}, & \text { for } t>t_{f},\end{cases}
$$

where

$$
\begin{aligned}
& t_{1}=\frac{2\left(L_{11}-L_{10}\right)}{v_{c}}, \\
& t_{2}=\frac{\left(L_{12}+L_{11}-2 L_{10}\right)}{v_{c}}, \\
& t_{f}=\frac{\left[2\left(L_{1 f}-L_{10}\right)+L_{11}-L_{12}\right]}{v_{c}} .
\end{aligned}
$$

In this section, the initial conditions are the same as those in Section 4.1, except for the fact that $L_{1}=5000 \mathrm{~km}$ and $\dot{L}_{1}=$ 0 are removed. They are replaced by the prescribed function shown in (47) to (48), where the constant velocity $v_{c}$ and the final position $L_{1 f}$ are specified as $0.5 \mathrm{~km} / \mathrm{s}$ and $4,628 \mathrm{~km}$, respectively. Since both formulations based on points $A$ and $C$ represent the same system, and the same initial state is considered, the numerical simulations by both formulations must be identical. Figure 8 shows distances $R_{A}$ and $R_{C}$ as functions to time. The results show that the distances periodically change, and one period is around $2.372 \times 10^{4}$ seconds. Besides, the mean values of $R_{A}$ and $R_{C}$ are $7.6706 \times$ $10^{4}$ and $8.1393 \times 10^{4} \mathrm{~km}$, respectively. Also, their amplitude is $3.4184 \times 10^{4}$ and $3.3896 \times 10^{4} \mathrm{~km}$, respectively. Figure 9 shows the true anomalies $\psi_{A}$ and $\psi_{C}$ as functions of time. The results show that they do not linearly change with time. To compare 


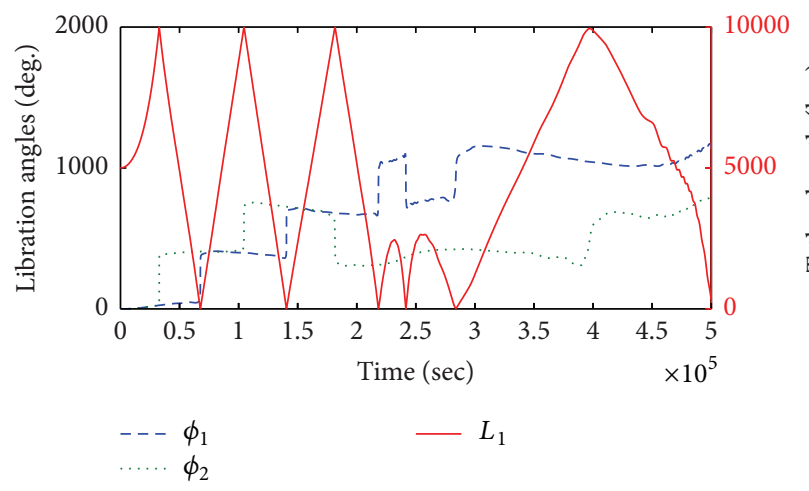

FIGURE 6: Libration angles and tether length $L_{1}$ by the formulations based on point $A$.

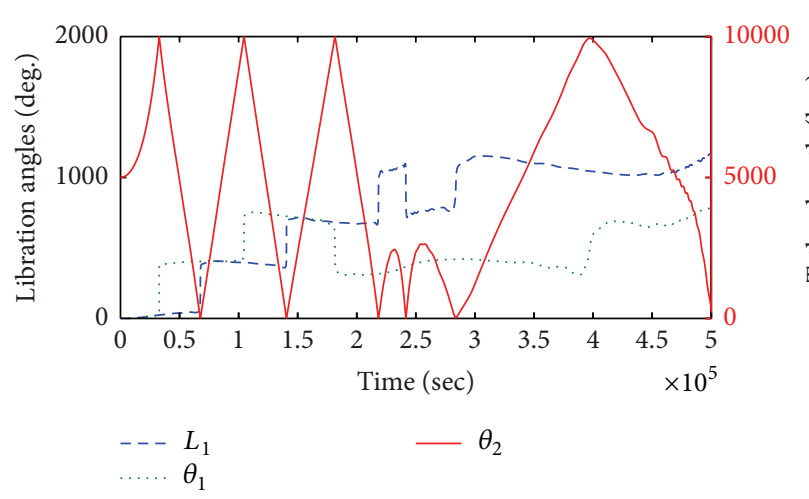

FIGURE 7: Libration angles and tether length $L_{1}$ by the formulations based on point $C$.

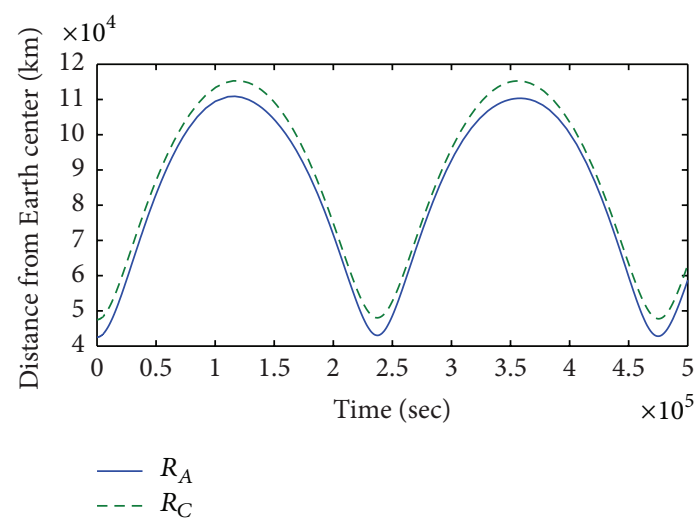

FIGURE 8: The distances from Earth center as function of time for a prescribed function $L_{1}(t)$.

the results with those in Section 4.1, all the values are similar, so the prescribed function does not affect $R_{A}$ and $R_{C}$ very much. Figure 10 shows libration angles $\phi_{1}$ and $\phi_{2}$ and tether length $L_{1}$ as functions of time. The results show that this prescribed function $L_{1}(t)$ yields the librations angles varying around between $\pm 50^{\circ}$. Figure 11 shows libration angles $\theta_{1}$ and $\theta_{2}$ as well as tether length $L_{1}$ as functions of time, and the results are similar to those shown in Figure 10.

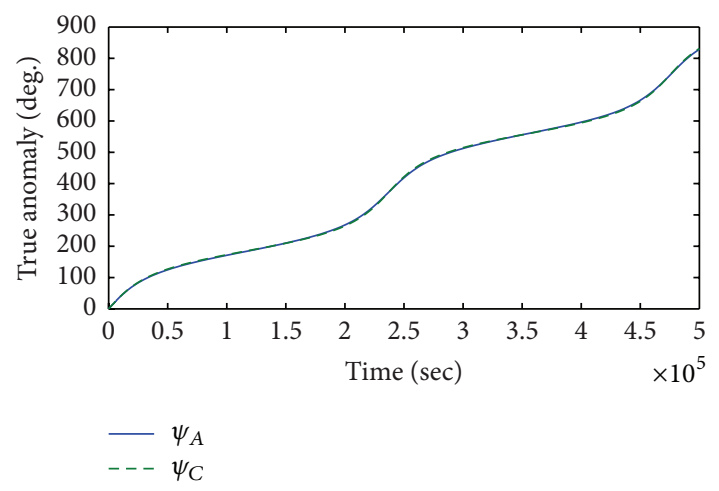

FIGURE 9: True anomaly as function of time for a prescribed function $L_{1}(t)$.

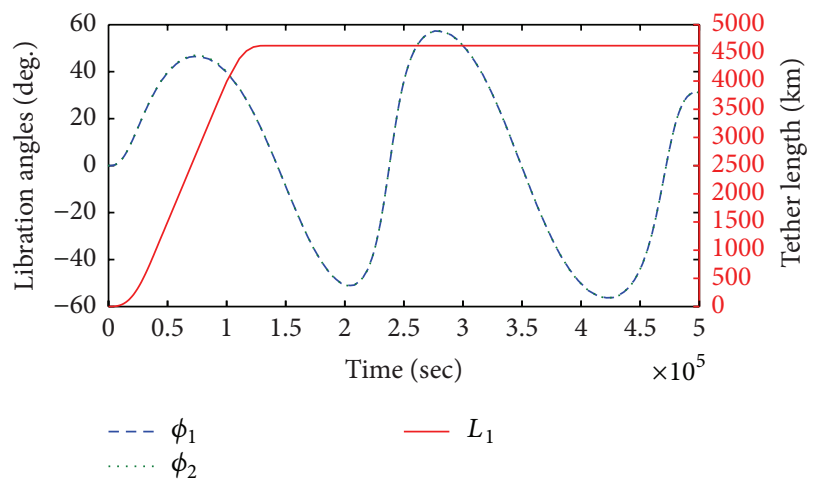

FIGURE 10: Libration angles and tether length $L_{1}$ by the formulations based on point $A$ for a prescribed function $L_{1}(t)$.

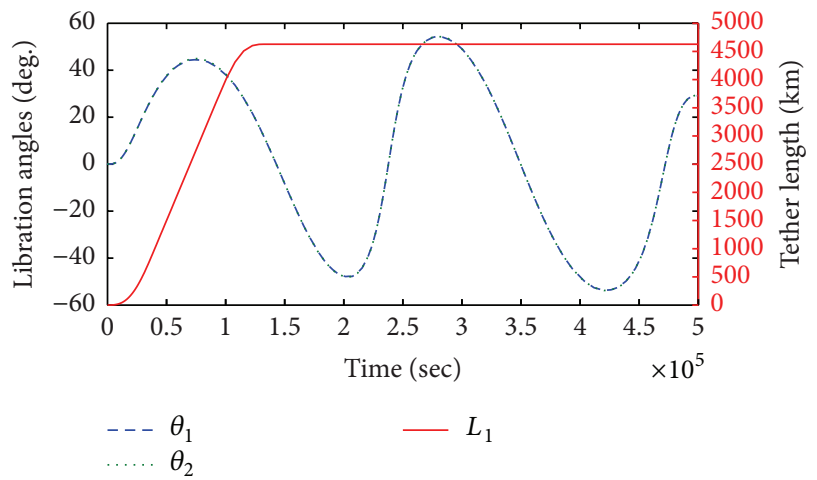

FIGURE 11: Libration angles and tether length $L_{1}$ by the formulations based on point $C$ with a prescribed function $L_{1}(t)$.

4.3. Free Libration with the Prescribed Function $L_{1}(t)$ on a Circular Orbit with a Constant Angular Rate. In this subsection, one considers that the tethered satellite system moves on a circular orbit, so the radius and the true anomaly become known parameters, and the system is governed by two ordinary differential equations. The tether length function $L_{1}(t)$ is the same as (47).

For the formulations based on point $A, R_{A}=42,522 \mathrm{~km}$, $\dot{\psi}_{A}=7.2923 \times 10^{-5}$, and $\dot{R}_{A}=\ddot{R}_{A}=\psi_{A}(0)=\ddot{\psi}_{A}=0$. 


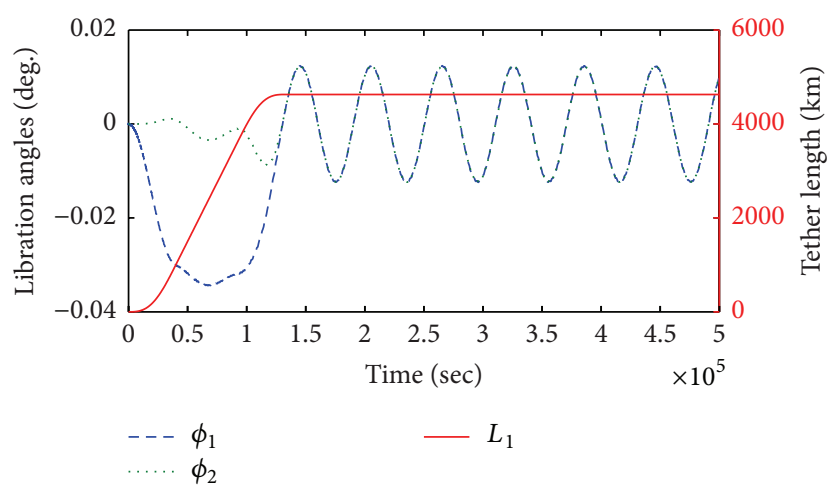

FIGURE 12: Libration angles $\phi_{1}(t)$ and $\phi_{2}(t)$ as functions of time based on the prescribed function $L_{1}(t)$ on a circular orbit with zero initial conditions.

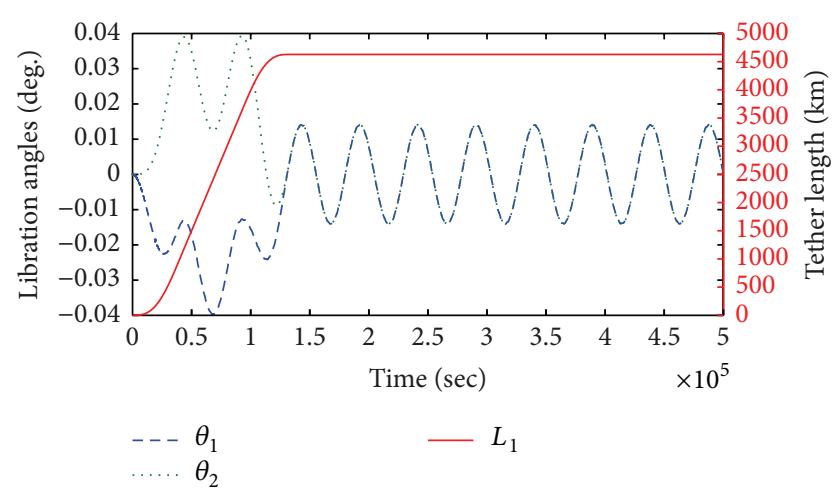

FIGURE 13: Libration angles $\theta_{1}(t)$ and $\theta_{2}(t)$ as functions of time based on the prescribed function $L_{1}(t)$ on a circular orbit with zero initial conditions.

The initial conditions are set as zeros. Figure 12 shows the libration angles $\phi_{1}(t)$ and $\phi_{2}(t)$ as functions of time based on the prescribed function $L_{1}(t)$ on a circular orbit. The results show that there is a transient response during the first $1.5 \times 10^{5}$ seconds, and the steady state shows that the libration angles oscillate around zero degrees with the amplitude of 0.01 degrees. For the formulations based on point $C, R_{C}=$ $42,522 \mathrm{~km}, \dot{\psi}_{C}=7.2923 \times 10^{-5}$, and $\dot{R}_{C}=\ddot{R}_{C}=\psi_{C}(0)=$ $\ddot{\psi}_{C}=0$. The initial conditions are also set as zeros. Note that the initial state is different from the former case, because the circular orbit is assigned to different points. Figure 13 shows the libration angles $\phi_{1}(t)$ and $\phi_{2}(t)$ as functions of time based on the prescribed function $L_{1}(t)$ on a circular orbit. The results show that there is a transient response during the first $1.5 \times 10^{5}$ seconds, and the steady state shows that the libration angles oscillate around zero degrees with the amplitude of 0.01 degrees.

The following simulations are similar to the former ones, except for the fact that the initial conditions are replaced by $\phi_{1}=\phi_{2}=\theta_{1}=\theta_{2}=0.1$ degrees. Based on the formulations based on point $A$, Figure 14 shows the libration angles $\phi_{1}(t)$ and $\phi_{2}(t)$ as functions of time based on the prescribed function $L_{1}(t)$ on a circular orbit. The results show that there is a transient response during the first $1.5 \times 10^{5}$ seconds,

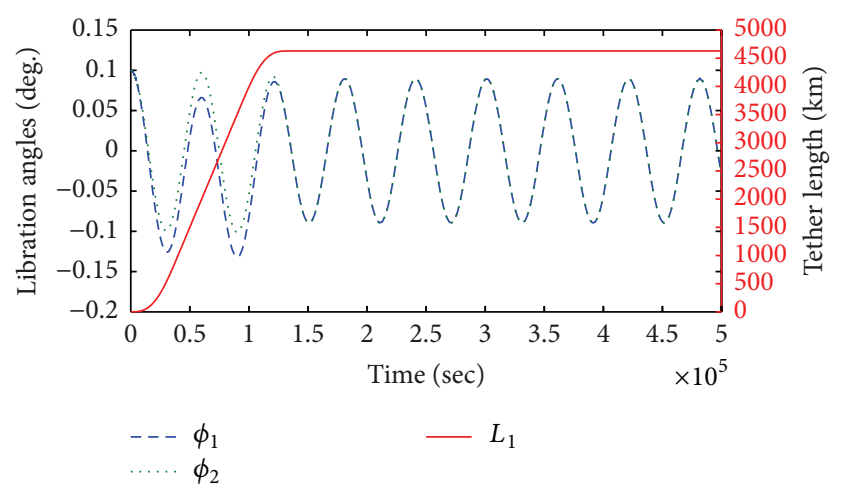

FIGURE 14: Libration angles $\phi_{1}(t)$ and $\phi_{2}(t)$ as functions of time based on the prescribed function $L_{1}(t)$ on a circular orbit.

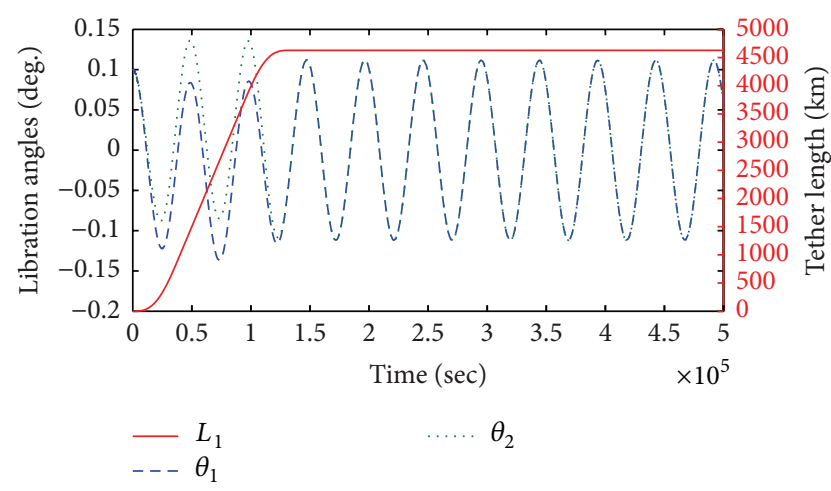

FIGURE 15: Libration angles $\theta_{1}(t)$ and $\theta_{2}(t)$ as functions of time based on the prescribed function $L_{1}(t)$ on a circular orbit.

and the steady state shows that the libration angles oscillate around zero degrees with the amplitude of 0.1 degrees. Based on the formulations based on point $C$, Figure 15 shows the libration angles $\phi_{1}(t)$ and $\phi_{2}(t)$ as functions of time based on the prescribed function $L_{1}(t)$ on a circular orbit. The results show that there is a transient response during the first $1.5 \times 10^{5}$ seconds, and the steady state shows that the libration angles oscillate around zero degrees with the amplitude of 0.1 degrees.

\section{Libration Suppression}

This section demonstrates the libration suppression by applying the SDRE control with a prescribed degree of stability, which is presented in Section 3.

5.1. Libration Control for the Equations of Motion Based on Point $A$. One considers that the tethered satellite system moves on a circular orbit with a constant rotating rate and satellite $D$ is fixedly located at the middle point of the tether. Thus, there are only two ordinary differential equations taken into account, and each equation is considered with a control torque, which is obtained based on the SDRE control method. 
Based on the formulations of the SDRE control, the two equations of motion are factorized as

$$
A^{(\phi)} \ddot{\phi}+B^{(\phi)} \dot{\phi}+C^{(\phi)} \phi=D u
$$

where $A^{(\phi)}, B^{(\phi)}$, and $C^{(\phi)}$ are $2 \times 2$ matrices, $D$ is a $2 \times 2$ identity matrix, $\phi=\left[\begin{array}{ll}\phi_{1} & \phi_{2}\end{array}\right]^{T}$, and $u=\left[\begin{array}{ll}T_{A} & T_{D}\end{array}\right]^{T}$. Besides, the components of the matrices and the vector are written as

$$
\begin{aligned}
& A_{11}^{(\phi)}=\left(m_{B}+m_{D}\right) L_{1}^{2}, \\
& A_{12}^{(\phi)}=A_{21}^{(\phi)}=m_{B} L_{1} L_{2} c_{12} \text {, } \\
& A_{22}^{(\phi)}=m_{B} L_{2}^{2} \text {, } \\
& B_{11}^{(\phi)}=2\left(m_{B}+m_{D}\right) L_{1} \dot{L}_{1} \text {, } \\
& B_{12}^{(\phi)}=-2 m_{D} L_{1} \dot{L}_{1} c_{12} \text {, } \\
& B_{21}^{(\phi)}=2 m_{D} L_{2} \dot{L}_{1} c_{12} \text {, } \\
& B_{22}^{(\phi)}=-2 m_{B} L_{2} \dot{L}_{1} \text {, } \\
& C_{11}^{(\phi)}=\left\{\mu m_{B} R_{B}^{-3}\left(-R_{A} L_{1} s_{1}+L_{1} L_{2} s_{1} c_{2}\right)\right. \\
& +\mu m_{D} R_{D}^{-3}\left(-R_{A} L_{1} s_{1}\right)+\left(m_{B}+m_{D}\right)\left[2 L_{1} \dot{L}_{1} \dot{\psi}_{A}\right. \\
& \left.+R_{A} L_{1} \dot{\psi}_{A}^{2}-L_{1} \ddot{R}_{A} s_{1}\right] \\
& \left.+m_{B}\left[L_{1} L_{2}\left(\dot{\psi}_{A}+\dot{\phi}_{2}\right)^{2} s_{1} c_{2}+L_{1} \ddot{L}_{1} s_{1} c_{2}\right]\right\}\left(\phi_{1}\right)^{-1}, \\
& C_{12}^{(\phi)}=\left\{\mu m_{B} R_{B}^{-3}\left(-L_{1} L_{2} c_{1} s_{2}\right)+\left(m_{B}+m_{D}\right)\right. \\
& \cdot\left[2 L_{1} \dot{R}_{A} \dot{\psi}_{A} c_{1}+\left(L_{1}^{2}+R_{A} L_{1} c_{1}\right) \ddot{\psi}_{A}\right] \\
& +m_{B}\left[-2 L_{1} \dot{L}_{1} \dot{\psi}_{A} c_{12}-L_{1} L_{2}\left(\dot{\psi}_{A}+\dot{\phi}_{2}\right)^{2} c_{1} s_{2}\right. \\
& \left.\left.+L_{1} L_{2} \ddot{\psi}_{A} c_{12}-L_{1} \ddot{L}_{1} c_{1} s_{2}\right]\right\}\left(\phi_{2}\right)^{-1} \text {, } \\
& C_{21}^{(\phi)}=\left\{\mu m_{B} R_{B}^{-3}\left(L_{1} L_{2} s_{1} c_{2}\right)+m_{B}\left[-2 L_{2} \dot{L}_{1} \dot{\psi}_{A} c_{12}\right.\right. \\
& +2 L_{2} \dot{R}_{A} \dot{\psi}_{A} c_{2}-L_{1} L_{2}\left(\dot{\psi}_{A}+\dot{\phi}_{1}\right)^{2} s_{1} c_{2} \\
& \left.\left.+\left(L_{2}^{2}+L_{1} L_{2} c_{12}+R_{A} L_{2} c_{2}\right) \ddot{\psi}_{A}+L_{2} \ddot{L}_{1} s_{1} c_{2}\right]\right\} \\
& \cdot\left(\phi_{1}\right)^{-1} \\
& C_{22}^{(\phi)}=\left\{\mu m_{B} R_{B}^{-3}\left(R_{A} L_{1} s_{2}-L_{1}^{2} c_{1} s_{2}\right)\right. \\
& +m_{B}\left[-2 L_{2} \dot{L}_{1} \dot{\psi}_{A}+R_{A} L_{2} \dot{\psi}_{A}^{2} s_{2}\right. \\
& \left.\left.+L_{1} L_{2}\left(\dot{\psi}_{A}+\dot{\phi}_{1}\right)^{2} c_{1} s_{2}-L_{2} \ddot{R}_{A} s_{2}-L_{2} \ddot{L}_{1} c_{1} s_{2}\right]\right\} \\
& \text { - }\left(\phi_{2}\right)^{-1} \text {. }
\end{aligned}
$$

The simulations are performed based on the following parameters: $R_{A}=42,522 \mathrm{~km}, \dot{\psi}_{A}=7.2923 \times 10^{-5} \mathrm{rad} / \mathrm{s}$, and $\dot{R}_{A}=\ddot{R}_{A}=\psi_{A}(0)=\ddot{\psi}_{A}=0$. Besides, the initial conditions are given as $\phi_{1}=1^{\circ}, \phi_{2}=-1^{\circ}$, and $\dot{\phi}_{1}=\dot{\phi}_{2}=0$. For simplicity, the weighting matrices in the SDRE control are selected as $Q=\operatorname{diag}(1,1,0,0)$ and $R=\operatorname{diag}(1,1)$. In order to demonstrate the performance of the prescribed degree of stability in the SDRE control, there are two cases tested as follows.

Case $1(\alpha=0)$. Figure 16 shows the libration angles as functions of time, and the results show that the SDRE control can suppress the librations around within 10 orbits. Although the time responses seemingly have high-frequency oscillations in the transient states (see Figure 16(a)), the oscillation period is around 1,930 seconds (see Figure 16(b)). Figure 17 shows the control torques as function of time, and the results show that the maximum control torque is around $0.16 \mathrm{MN}-\mathrm{m}$.

Case $2\left(\alpha=10^{-6}\right)$. Seemingly, the convergence rate for $\alpha=0$ is little slow, so this test case is based on $\alpha=10^{-6}$. Figure 18 shows the libration angles as functions of time, and the results show that the SDRE control can suppress the librations around within 8 orbits, which indicates that the proposed SDRE control with a prescribed degree of stability can effectively reduce the converge time. This figure also shows a high-frequency oscillation in the transient state, and the oscillation period is the same as 1,930 seconds. Figure 19 shows the control torques as function of time, and the results show that the maximum control torque is around $0.28 \mathrm{MN}$ $\mathrm{m}$, which is larger than those presented in previous case. This is the penalty to reduce the convergence time.

5.2. Libration Control for the Equations of Motion Based on Point C. The same orbit and orbital rate are considered in this subsection, and satellite $D$ is also fixedly located at the middle point of the tether. Thus, there are still only two ordinary differential equations taken into account, and each equation is considered with a control torque, which will be determined by applying the SDRE control method.

Based on the formulations of the SDRE control, the two equations of motion are factorized as

$$
A^{(\theta)} \ddot{\theta}+B^{(\theta)} \dot{\theta}+C^{(\theta)} \theta=D u
$$

where $A^{(\theta)}, B^{(\theta)}$, and $C^{(\theta)}$ are $2 \times 2$ matrices and $\theta$ refers to $\theta=\left[\begin{array}{ll}\theta_{1} & \theta_{2}\end{array}\right]^{T}$. Besides, the components of the matrices and the vector are written as

$$
\begin{aligned}
& A_{11}^{(\theta)}=a_{1} L_{1}^{2}, \\
& A_{12}^{(\theta)}=A_{21}^{(\theta)}=a_{3} L_{1} L_{2} c_{12}, \\
& A_{22}^{(\theta)}=a_{2} L_{2}^{2}, \\
& B_{11}^{(\theta)}=2 a_{1} L_{1} \dot{L}_{1}, \\
& B_{12}^{(\theta)}=-2 a_{3} L_{1} \dot{L}_{1} c_{12},
\end{aligned}
$$




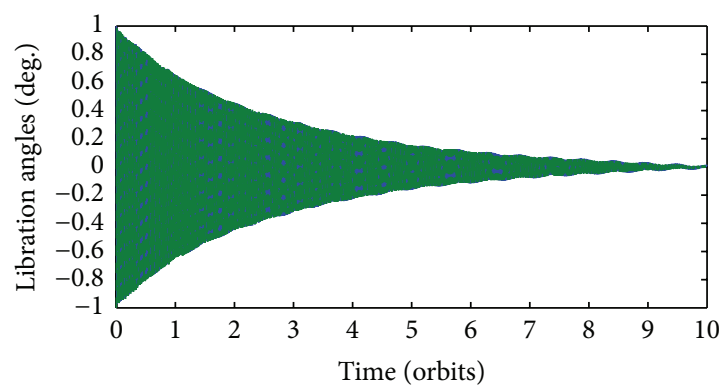

$-\phi_{1}$
$--\phi_{2}$

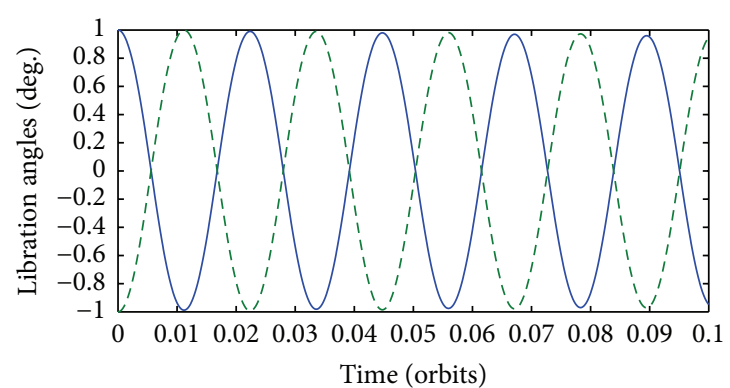

$-\phi_{1}$

(a)

(b)

FIGURE 16: Libration angles from the formulations based on point $A$ by applying the SDRE control with $\alpha=0$ for (a) the first 10 orbits and (b) the first 0.1 orbits.

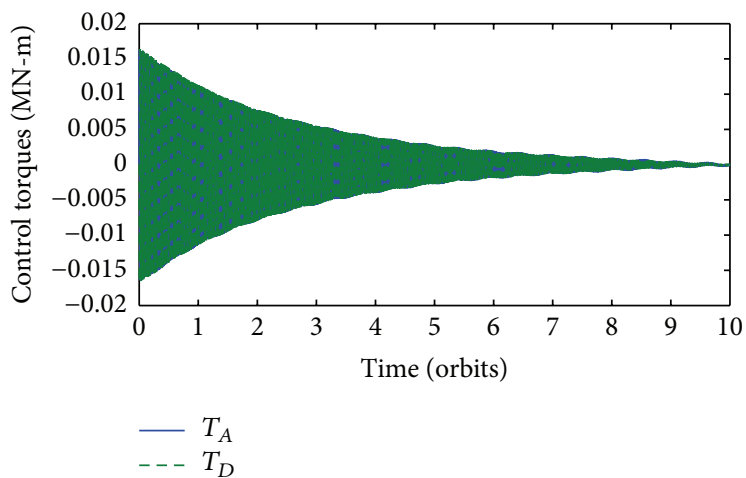

(a)

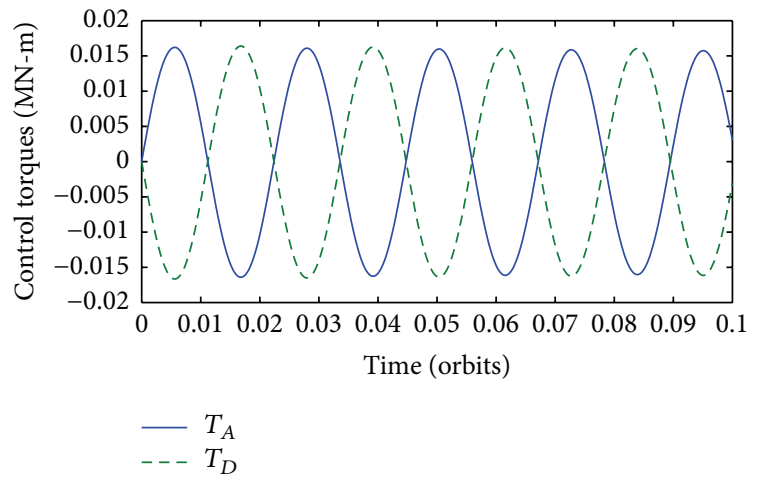

(b)

FIGURE 17: Control torques from the formulations based on point $A$ by applying the SDRE control with $\alpha=0$ for (a) the first 10 orbits and (b) the first 0.1 orbits.

$$
\begin{aligned}
B_{21}^{(\theta)} & =2 a_{3} L_{2} \dot{L}_{1} c_{12}, \\
B_{22}^{(\theta)} & =-2 a_{2} L_{2} \dot{L}_{1}, \\
C_{11}^{(\theta)} & =\left\{\mu m _ { A } R _ { A } ^ { - 3 } \left[\left(1-\rho_{A}\right) R_{C} L_{1} s_{1}\right.\right. \\
& \left.-\left(1-\rho_{A}\right) \rho_{B} L_{1} L_{2} s_{1} c_{2}\right]+\mu m_{B} R_{B}^{-3}\left[-\rho_{A} R_{C} L_{1} s_{1}\right. \\
& \left.-\rho_{A}\left(1-\rho_{B}\right) L_{1} L_{2} s_{1} c_{2}\right]+\mu m_{D} R_{D}^{-3}\left[-\rho_{A} R_{C} L_{1} s_{1}\right. \\
& \left.+\rho_{A} \rho_{B} L_{1} L_{2} s_{1} c_{2}\right]+2 a_{1} L_{1} \dot{L}_{1} \dot{\psi}_{C}+a_{3}\left[L_{1} \ddot{L}_{1} s_{1} c_{2}\right. \\
& \left.\left.+L_{1} L_{2}\left(\dot{\psi}_{C}+\dot{\theta}_{2}\right)^{2} s_{1} c_{2}\right]\right\}\left(\theta_{1}\right)^{-1}, \\
C_{12}^{(\theta)} & =\left\{\mu m_{A} R_{A}^{-3}\left(1-\rho_{A}\right) \rho_{B} L_{1} L_{2} c_{1} s_{2}+\mu m_{B} R_{B}^{-3} \rho_{A}(1\right. \\
& \left.-\rho_{B}\right) L_{1} L_{2} c_{1} s_{2}-\mu m_{D} R_{D}^{-3} \rho_{A} \rho_{B} L_{1} L_{2} c_{1} s_{2} \\
& +a_{3}\left[-L_{1} \ddot{L}_{1} c_{1} s_{2}-L_{1} L_{2}\left(\dot{\psi}_{C}+\dot{\theta}_{2}\right)^{2} c_{1} s_{2}\right. \\
& \left.\left.-2 L_{1} \dot{L}_{1} \dot{\psi}_{C} c_{12}\right]\right\}\left(\theta_{2}\right)^{-1},
\end{aligned}
$$

$$
\begin{aligned}
& C_{21}^{(\theta)}=\left\{\mu m_{A} R_{A}^{-3}\left[\rho_{B} R_{C} L_{2} s_{2}+\left(1-\rho_{A}\right) \rho_{B} L_{1} L_{2} s_{1} c_{2}\right]\right. \\
& +\mu m_{B} R_{B}^{-3}\left[-\left(1-\rho_{B}\right) R_{C} L_{2} s_{2}\right. \\
& \left.+\rho_{A}\left(1-\rho_{B}\right) L_{1} L_{2} s_{1} c_{2}\right]+\mu m_{D} R_{D}^{-3}\left[\rho_{B} R_{C} L_{2} s_{2}\right. \\
& \left.\quad-\rho_{A} \rho_{B} L_{1} L_{2} s_{1} c_{2}\right]+a_{3}\left[L_{2} \ddot{L}_{1} s_{1} c_{2}\right. \\
& \left.\left.\quad-L_{1} L_{2}\left(\dot{\psi}_{C}+\dot{\theta}_{1}\right)^{2} s_{1} c_{2}+2 L_{2} \dot{L}_{1} \dot{\psi}_{C} c_{12}\right]\right\}\left(\theta_{1}\right)^{-1}, \\
& C_{22}^{(\theta)}=\left\{-\mu m_{A} R_{A}^{-3}\left(1-\rho_{A}\right) \rho_{B} L_{1} L_{2} c_{1} s_{2}\right. \\
& \quad-\mu m_{B} R_{B}^{-3} \rho_{A}\left(1-\rho_{B}\right) L_{1} L_{2} c_{1} s_{2} \\
& +\mu m_{D} R_{D}^{-3} \rho_{A} \rho_{B} L_{1} L_{2} c_{1} s_{2}-2 a_{2} L_{2} \dot{L}_{1} \dot{\psi}_{C} \\
& \left.\quad+a_{3}\left[-L_{2} \ddot{L}_{1} c_{1} s_{2}+L_{1} L_{2}\left(\dot{\psi}_{C}+\dot{\theta}_{1}\right)^{2} c_{1} s_{2}\right]\right\}\left(\theta_{2}\right)^{-1} .
\end{aligned}
$$

In order to compare the simulations with those in previous subsection, the initial conditions are the same, so 


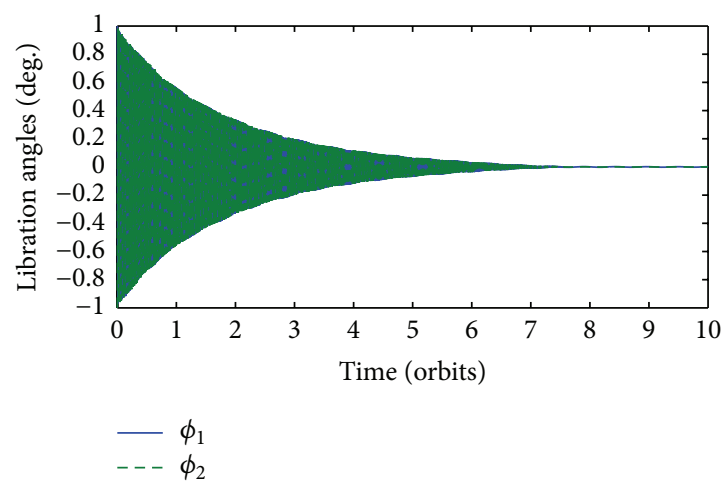

(a)

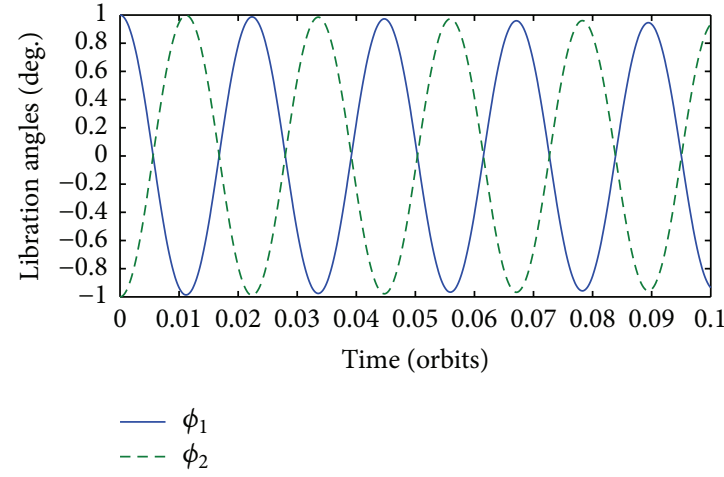

(b)

FIGURE 18: Libration angles from the formulations based on point $A$ by applying the SDRE control with $\alpha=10^{-6}$ for (a) the first 10 orbits and (b) the first 0.1 orbits.

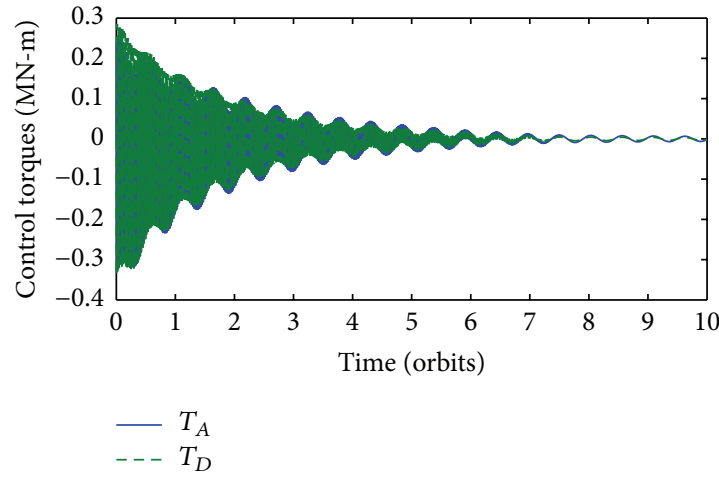

(a)

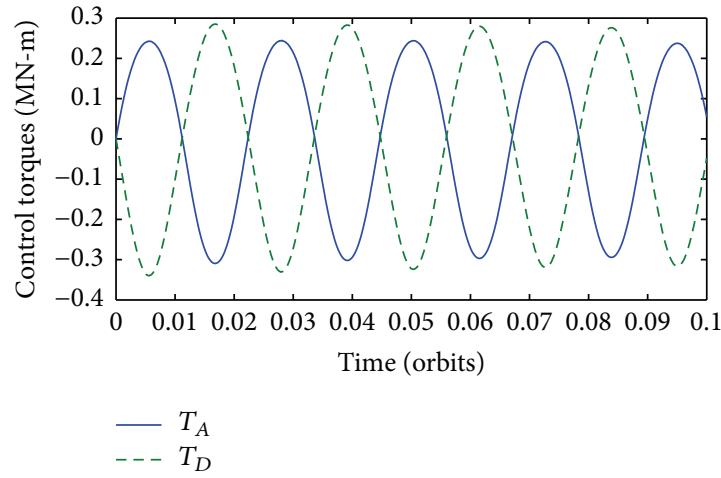

(b)

FIGURE 19: Control torques from the formulations based on point $A$ by applying the SDRE control with $\alpha=10^{-6}$ for (a) the first 10 orbits and (b) the first 0.1 orbits.

the initial libration angles are given as $\theta_{1}(0)=0.9649^{\circ}$ and $\theta_{2}(0)=-1.0351^{\circ}$. Based on the initial condition, the orbital radius is given as $R_{C}=47,521 \mathrm{~km}$, and the orbital rate is the same as $\dot{\psi}_{C}=7.2923 \times 10^{-5} \mathrm{rad} / \mathrm{s}$. Besides, the parameters are given as $\dot{R}_{C}=\ddot{R}_{C}=\psi_{C}(0)=\ddot{\psi}_{C}=0$. In the SDRE control, the weighting matrices are the same as $Q=\operatorname{diag}(1,1,0,0)$ and $R=\operatorname{diag}(1,1)$. Note that the weighting matrices have different meaning due to different state variables. Thus, although most of parameters are the same as those in previous subsection, the simulation results will be different. Similarly, two cases are tested to demonstrate the prescribed degree of stability.

Case $3(\alpha=0)$. Figure 20 shows the libration angles as functions of time, and the results show that the SDRE control can suppress the librations around within 10 orbits. Although the time responses seemingly have high-frequency oscillations in the transient states (see Figure 20(a)), the oscillation period is around 3,954.8 seconds (see Figure 20(b)), which is almost twice time in Figure 16(b). Figure 21 shows the control torques as function of time, and the results show that the maximum control torque is around $0.35 \mathrm{MN}-\mathrm{m}$, which is also larger than the torque in Figure 16.
Case $4\left(\alpha=10^{-6}\right)$. Seemingly, the convergence rate for $\alpha=0$ is little slow, so this test case is based on $\alpha=10^{-6}$. Figure 22 shows the libration angles as functions of time, and the results show that the SDRE control can suppress the librations around within 8 orbits, which indicates that the proposed SDRE control with a prescribed degree of stability can effectively reduce the converge time. This figure also shows a high-frequency oscillation in the transient state, and the oscillation period is the same as 3,954.8 seconds. Figure 23 shows the control torques as function of time, and the results show that the profile is similar to that in Case 1, and the difference is that the torque converges faster.

\section{Conclusions}

This paper investigates the nonlinear dynamic analysis and control of a tethered satellite system. First, the system is modeled by a two-piece dumbbell. There are three satellites in the system, and each satellite is assumed to be a point mass. A constant-length massless tether connects the three satellites. Based on the model, two sets of equations of motion are derived, which are based on the first satellite 


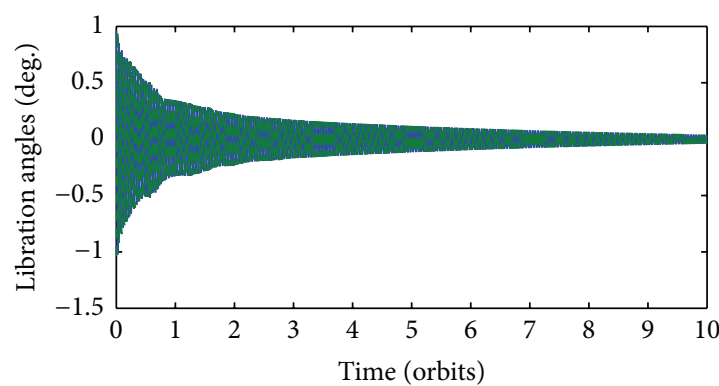

$-\theta_{1}$

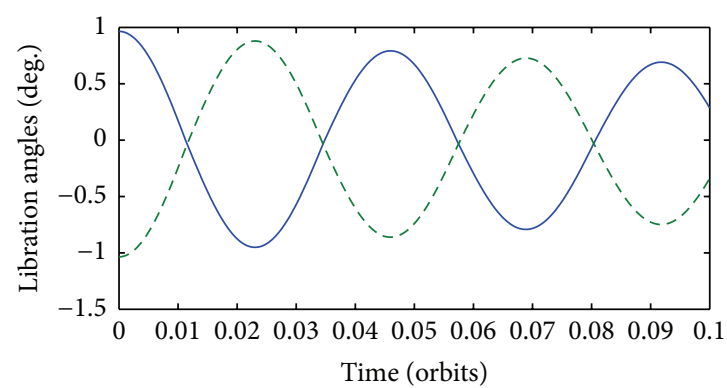

$-\theta_{1}$

(a)

(b)

Figure 20: Libration angles from the formulations based on point $C$ by applying the SDRE control with $\alpha=0$ for (a) the first 10 orbits and (b) the first 0.1 orbits.

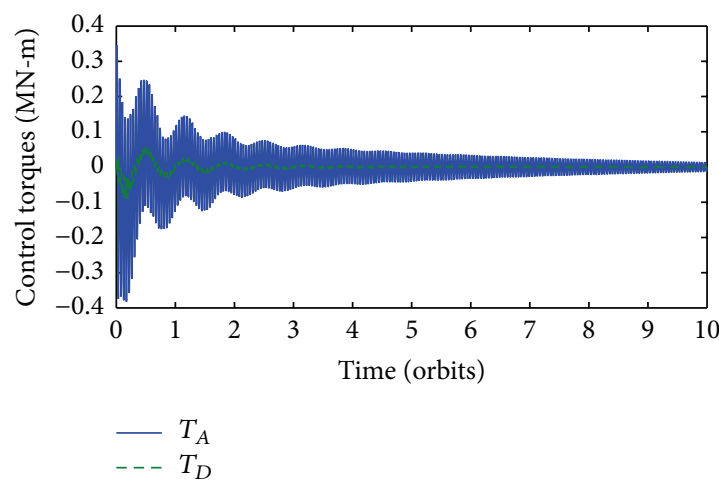

(a)

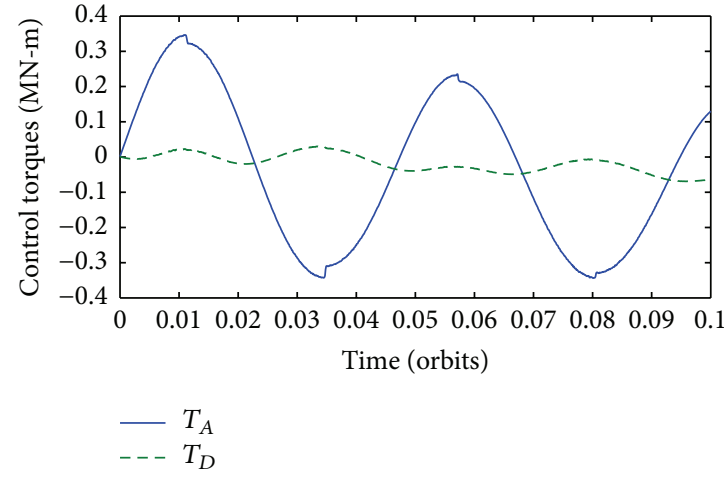

(b)

FIGURE 21: Control torques from the formulations based on point $C$ by applying the SDRE control with $\alpha=0$ for (a) the first 10 orbits and (b) the first 0.1 orbits.

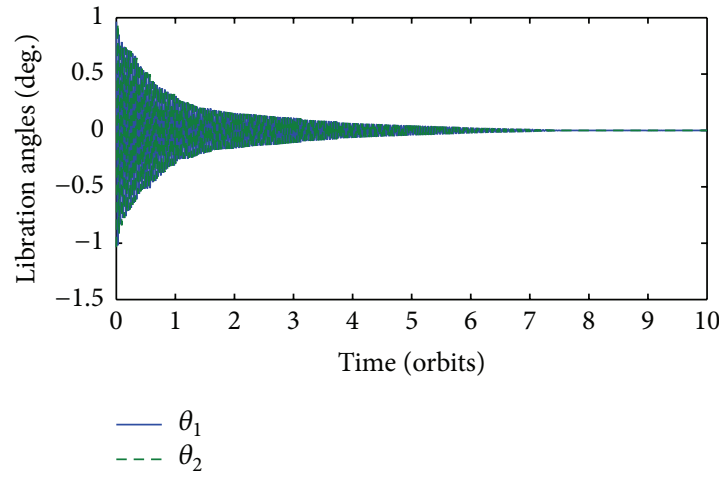

(a)

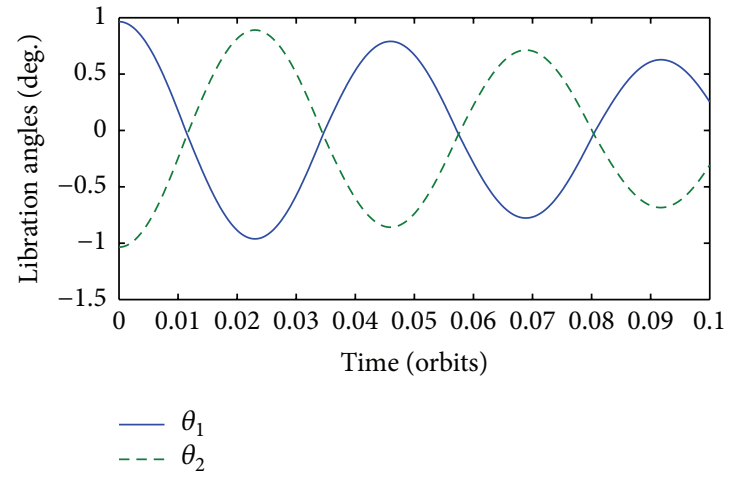

(b)

FIGURE 22: Libration angles from the formulations based on point $C$ by applying the SDRE control with $\alpha=10^{-6}$ for (a) the first 10 orbits and (b) the first 0.1 orbits.

and the mass center of the system. Secondly, the SDRE with a prescribed degree of stability is proposed, which can reduce the convergence time of a nonlinear system. Thirdly, the nonlinear dynamic analysis is performed by numerical simulations. The results show that the librations of the system affected the motion of the middle satellite. Also, its motion affects the orbital radius and orbital rate. Finally, the proposed SDRE approach is developed and is applied to the system, 


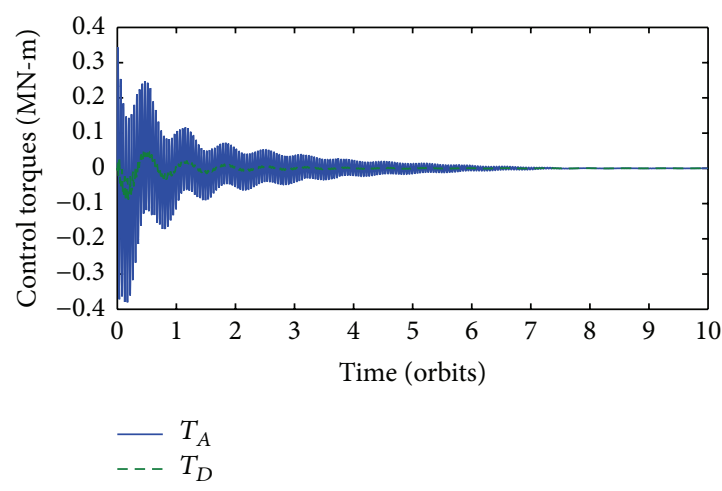

(a)

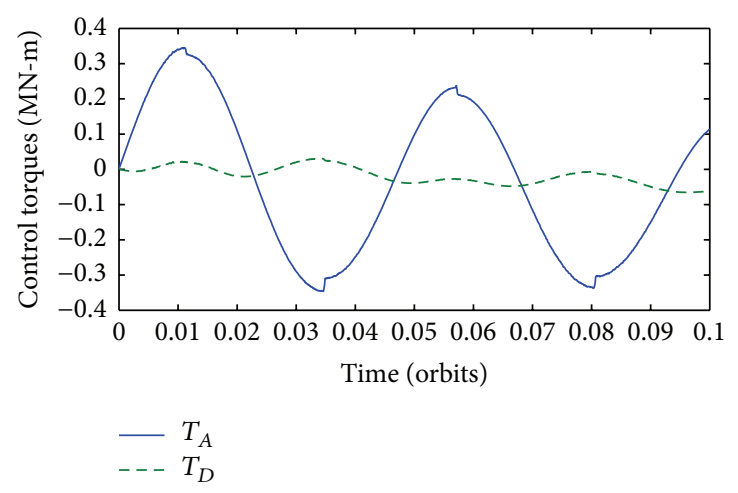

(b)

FIGURE 23: Control torques from the formulations based on point $C$ by applying the SDRE control with $\alpha=10^{-6}$ for (a) the first 10 orbits and (b) the first 0.1 orbits.

and the numerical examples demonstrate the efficiency of the proposed approach in the system.

\section{Competing Interests}

The author declares that they have no competing interests.

\section{Acknowledgments}

The study was sponsored by a Grant, MOST 103-2221-E-011028, from the Ministry of Science and Technology, Taiwan.

\section{References}

[1] K. E. Tsiolkovsky, Speculations Between Earth and Sky, Isd-vo AN-SSSR, 1895, (reprinted in 1959).

[2] G. Colombo, E. M. Goposchkin, M. D. Grossi, and G. C. Weiffenbach, "Shuttle-borne skyhook: a new tool for low-orbitalaltitude research," Contract NAS8-02138, Smithsonian Institution Astrophysical Observatory Report, Cambridge, Mass, USA, 1974.

[3] M. L. Cosmo and E. C. Lorenzini, Tethers in Space Handbook, NASA Marshall Space Flight Center, Huntsville, Ala, USA, 3rd edition, 1997.

[4] Y. He, B. Liang, and W. Xu, "Study on the stability of Tethered Satellite System," Acta Astronautica, vol. 68, no. 11-12, pp. 19641972, 2011.

[5] K. Nakanishi, H. Kojima, and T. Watanabe, "Trajectories of inplane periodic solutions of tethered satellite system projected on van der Pol planes," Acta Astronautica, vol. 68, no. 7-8, pp. 1024-1030, 2011.

[6] H. Kojima, Y. Furukawa, and P. M. Trivailo, "Experimental study on delayed feedback control for libration of tethered satellite system," Journal of Guidance, Control, and Dynamics, vol. 35, no. 3, pp. 998-1002, 2012.

[7] W. Zhang, F. B. Gao, and M. H. Yao, "Periodic solutions and stability of a tethered satellite system," Mechanics Research Communications, vol. 44, pp. 24-29, 2012.

[8] H. Hu, H. Wen, and D. P. Jin, "Dynamic control and groundbased experiments of a tethered satellite system," in IUTAM Symposium on Nonlinear Dynamics for Advanced Technologies and Engineering Design: Proceedings of the IUTAM Symposium on Nonlinear Dynamics for Advanced Technologies and Engineering Design, held Aberdeen, UK, 27-30 July 2010, vol. 32 of IUTAM Bookseries, pp. 375-386, Springer, Amsterdam, The Netherlands, 2013.

[9] W. Jung, A. P. Mazzoleni, and J. Chung, "Dynamic analysis of a tethered satellite system with a moving mass," Nonlinear Dynamics, vol. 75, no. 1-2, pp. 267-281, 2014.

[10] W. Jung, A. P. Mazzoleni, and J. Chung, "Nonlinear dynamic analysis of a three-body tethered satellite system with deployment/retrieval," Nonlinear Dynamics, vol. 82, no. 3, pp. 11271144, 2015.

[11] A. A. T. Hong and R. Varatharajoo, "Dynamics of a flexible tethered satellite system utilising various materials for coplanar and non-coplanar models," Advances in Space Research, vol. 56, no. 4, pp. 648-663, 2015.

[12] A. A. T. Hong, R. Varatharajoo, and F. Romli, "Analytic solutions for Tethered Satellite System (TSS) subject to internal and external torques undergoing a spin-up and spin-down maneuvers," Aircraft Engineering and Aerospace Technology, vol. 87, no. 6, pp. 514-520, 2015.

[13] A. K. Misra and V. J. Modi, "Dynamics and control of tether connected two-body systems-a brief review," in Proceedings of the Paris International Astronautical Federation Congress, vol. 1, Paris, France, 1982.

[14] M. P. Cartmell and D. J. McKenzie, "A review of space tether research," Progress in Aerospace Sciences, vol. 44, no. 1, pp. 1-21, 2008.

[15] P. Williams, "A review of space tether technology," Recent Patents on Space Technology, vol. 2, no. 1, pp. 22-36, 2012.

[16] Y. Chen, R. Huang, X. Ren, L. He, and Y. He, "History of the tether concept and tether missions: a review," ISRN Astronomy and Astrophysics, vol. 2013, Article ID 502973, 7 pages, 2013.

[17] Y. Chen, R. Huang, L. He, X. Ren, and B. Zheng, "Dynamical modelling and control of space tethers: a review of space tether research," Nonlinear Dynamics, vol. 77, no. 4, pp. 1077-1099, 2014.

[18] J. D. Pearson, "Approximation methods in optimal control I. Sub-optimal control," Journal of Electronics and Control, vol. 13, no. 5, pp. 453-469, 1962.

[19] J. R. Coultier, C. N. Souza, and C. P. Mracek, "Nonlinear regulation and nonlinear $\mathrm{H}$, control via the state-dependent Riccati 
equation technique. Part 1: theory; Part 2: examples," in Proceedings of the International Conference on Nonlinear Problems in Aviation and Aerospace, pp. 117-141, Daytona Beach, Fla, USA, May 1996.

[20] X. Zhao and F. Deng, "Solution of the HJI equations for nonlinear $H_{\infty}$ control design by state-dependent Riccati equations approach," Journal of Systems Engineering and Electronics, vol. 22, no. 4, pp. 654-660, 2011.

[21] S. R. Nekoo, "Nonlinear closed loop optimal control: a modified state-dependent Riccati equation," ISA Transactions, vol. 52, no. 2, pp. 285-290, 2013.

[22] I. Chang and J. Bentsman, "Constrained discrete-time statedependent Riccati equation technique: a model predictive control approach," in Proceedings of the 52nd IEEE Conference on Decision and Control (CDC 2013), pp. 5125-5130, Firenze, Italy, December 2013.

[23] N. Babaei and M. U. Salamci, "State dependent riccati equation based model reference adaptive control design for nonlinear systems," in Proceedings of the 24th International Conference on Information, Communication and Automation Technologies (ICAT '13), pp. 1-8, IEEE, Sarajevo, Bosnia and Herzegovina, November 2013.

[24] S. R. Nekoo and B. Geranmehr, "Nonlinear observer-based optimal control using the state-dependent Riccati equation for a class of non-affine control systems," Control Engineering and Applied Informatics, vol. 16, no. 2, pp. 5-13, 2014.

[25] X. Wang, J. Long, W. Sun, and W. Lian, “The generalized state dependent riccati equation control of continuous time nonlinear systems," in Proceedings of the International Mechanical Engineering Congress and Exposition (ASME '14), American Society of Mechanical Engineers, 2014.

[26] M. H. Korayem and S. R. Nekoo, "Nonlinear optimal control via finite time horizon state-dependent Riccati equation," in Proceedings of the 2nd RSI/ISM International Conference on Robotics and Mechatronics (ICRoM 2014), pp. 878-883, Tehran, Iran, October 2014.

[27] M. H. Korayem and S. R. Nekoo, "Finite-time state-dependent Riccati equation for time-varying nonaffine systems: rigid and flexible joint manipulator control," ISA Transactions, vol. 54, pp. 125-144, 2015.

[28] J. R. Cloutier, "State-dependent Riccati equation techniques: an overview," in Proceedings of the American Control Conference, vol. 2, pp. 932-936, Albuquerque, NM, USA, June 1997.

[29] J. R. Cloutier and D. T. Stansbery, "The capabilities and art of State-dependent Riccati equation-based design," in Proceedings of the American Control Conference, vol. 1, pp. 86-91, May 2002.

[30] T. Cimen, "State-dependent Riccati equation (SDRE) control: a survey," in Proceedings of the 17th World Congress of the International Federation of Automatic Control (IFAC '08), pp. 611, Seoul, Republic of Korea, July 2008.

[31] T. Çimen, "Systematic and effective design of nonlinear feedback controllers via the state-dependent Riccati equation (SDRE) method," Annual Reviews in Control, vol. 34, no. 1, pp. 32-51, 2010.

[32] T. Çimen, "Survey of state-dependent Riccati equation in nonlinear optimal feedback control synthesis," Journal of Guidance, Control, and Dynamics, vol. 35, no. 4, pp. 1025-1047, 2012. 


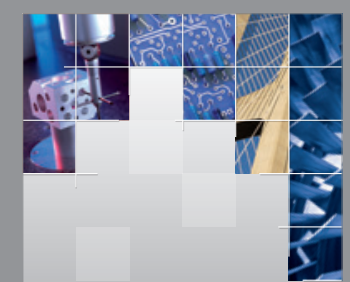

\section{Enfincering}
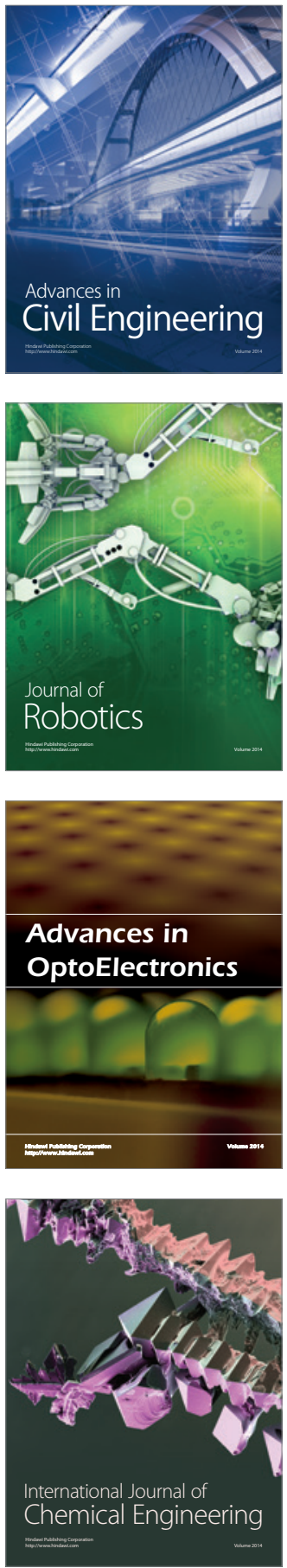

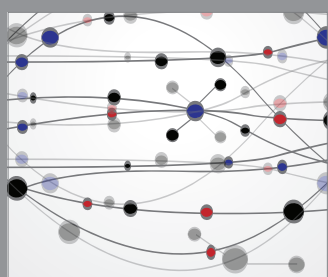

The Scientific World Journal

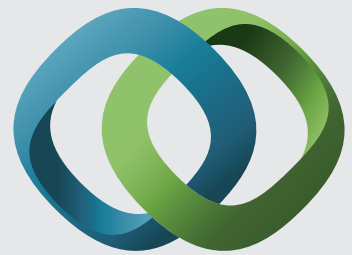

\section{Hindawi}

Submit your manuscripts at

http://www.hindawi.com
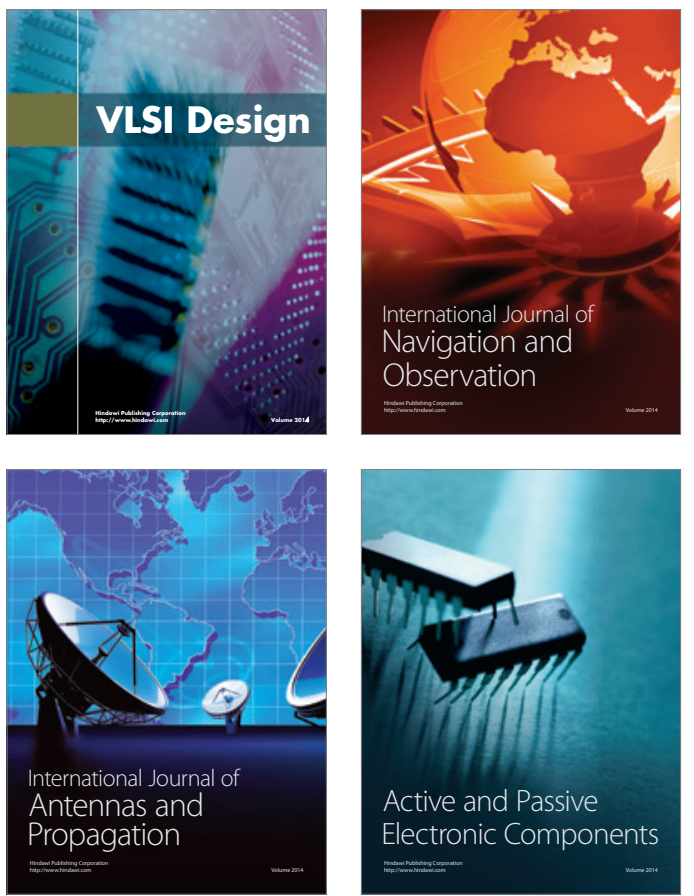
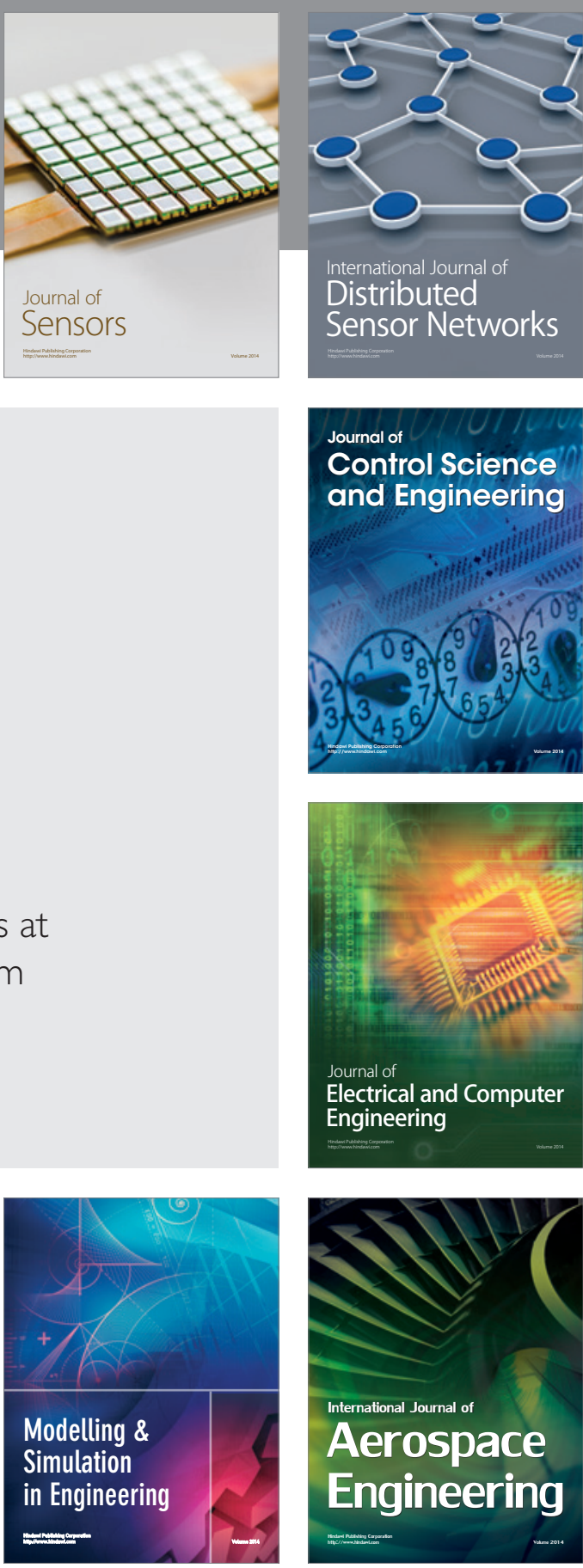

International Journal of

Distributed

Sensor Networks

Journal of

Control Science

and Engineering
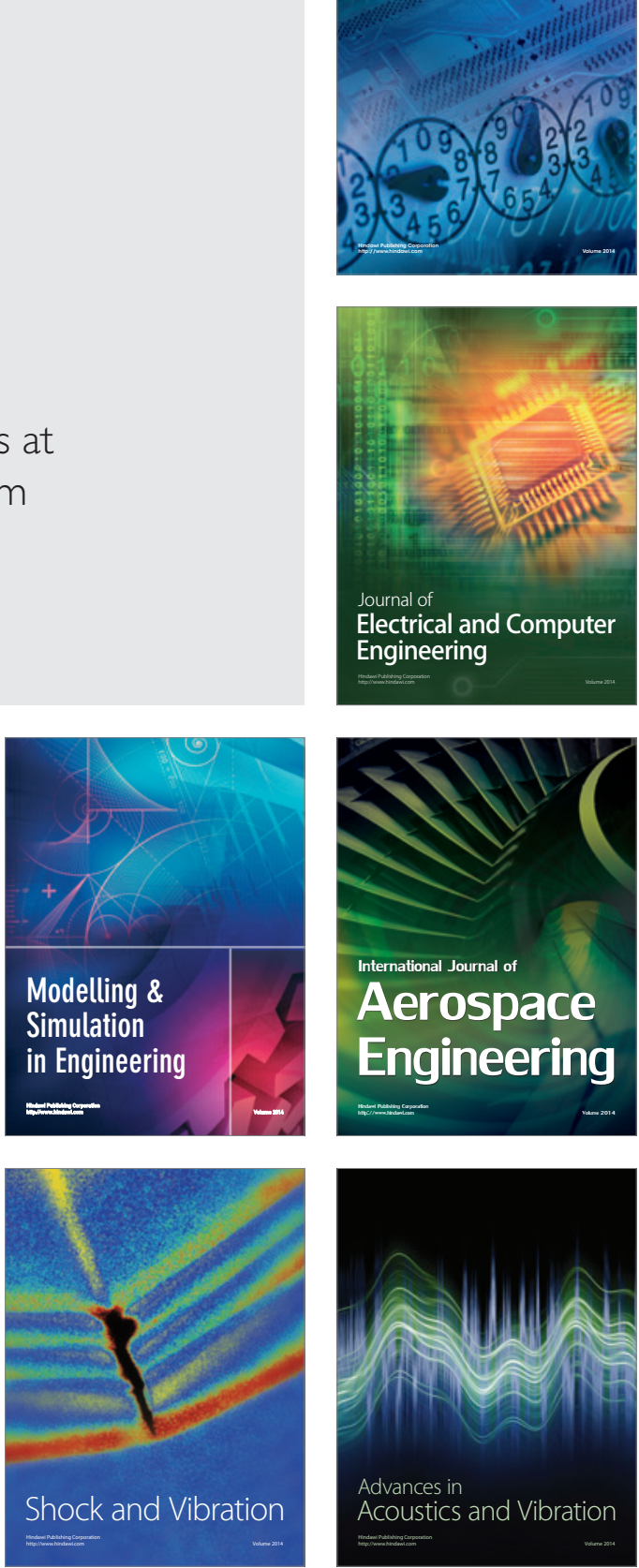